\title{
Luminescent Osmium(II) Complexes with Functionalized 2-Phenylpyridine Chelating Ligands: Preparation, Structural Analyses, and Photophysical Properties
}

\author{
Kwun-Chi Hwang, Jing-Lin Chen, and Yun Chi* \\ Department of Chemistry, National Tsing Hua University, Hsinchu 300, Taiwan \\ Chun-Wei Lin, Yi-Ming Cheng, Gene-Hsiang Lee, and Pi-Tai Chou* \\ Department of Chemistry, National Taiwan University, Taipei 106, Taiwan
}

Sue-Yi Lin and Ching-Fong Shu*

Department of Applied Chemistry, National Chiao Tung University, Hsinchu 300, Taiwan

Received November 25, 2007

\begin{abstract}
Cyclometalated osmium complexes with the formulas $\left[\mathrm{Os}(\mathrm{ppy})_{2}(\mathrm{CO})_{2}\right](\mathbf{1 a}, \mathbf{b}),\left[\mathrm{Os}(\mathrm{dfppy})_{2}(\mathrm{CO})_{2}\right](\mathbf{2 a}, \mathbf{b})$, and $\left[\mathrm{Os}(\mathrm{btfppy})_{2}(\mathrm{CO})_{2}\right](3 \mathbf{a}, \mathbf{b})$ have been synthesized, for which the chelating chromophores ppyH, dfppyH, and btfppyH denote 2-phenylpyridine, 2-(2,4-difluorophenyl)pyridine, and 2-(2,4-bis(trifluoromethyl)phenyl)pyridine, respectively. The isomers $1 \mathbf{a}-\mathbf{3 a}$, possessing an intrinsic $C_{2}$ rotational axis as determined by single-crystal $\mathrm{X}$-ray diffraction analysis, underwent slow isomerization in solution at elevated temperature, giving the respective thermodynamic products $1 b-3 b$, which showed a distinctive coordination arrangement produced by a $180^{\circ}$ rotation of one cyclometalated ligand around the Os(II) metal center. In contrast to the case for $\mathbf{1 a , b}$ and $\mathbf{2 a}$, which are inert to substitution, complexes $\mathbf{2 b}$ and $\mathbf{3 b}$ (or $\mathbf{3 a}$ ) readily react with $\mathrm{PPh}_{2} \mathrm{Me}$ to afford the products [Os(dfppy) $(\mathrm{CO})\left(\mathrm{PPh}_{2} \mathrm{Me}\right)$ ] (4) and [Os(btfppy $\left.\left.)_{2}\right)\left(\mathrm{PPh}_{2} \mathrm{Me}\right)\right]$ (6), in which the incoming $\mathrm{PPh}_{2} \mathrm{Me}$ replaced the $\mathrm{CO}$ located trans to the carbon atom of one cyclometalated ligand. UV-vis and emission spectra were measured, revealing the lowest excited state for all complexes as a nominally ligand-centered ${ }^{3} \pi \pi^{*}$ state mixed with certain MLCT character. Introduction of the electron-withdrawing substituents on the cyclometalated chelates or replacement of one CO ligand with phosphine at the metal center increased the MLCT contribution in the first excited state, giving a broad and featureless emission with greatly enhanced quantum yields.
\end{abstract}

\section{Introduction}

Cyclometalated chelates represent an important class of nitrogen heterocyclic ligands for transition-metal coordination chemistry. ${ }^{1}$ The major division in this classification is according to reaction pattern; one such reaction is that an aromatic segment, cf. a phenyl group of a chelating molecule

* To whom correspondence should be addressed. E-mail: ychi@ mx.nthu.edu.tw (Y.C.); chop@ntu.edu.tw, (P.-T.C.); shu@cc.nctu.edu.tw (C.-F.S.).

(1) (a) Babudri, F.; Farinola, G. M.; Naso, F.; Ragni, R. Chem. Commun. 2007, 1003. (b) Mohr, F.; Priver, S. H.; Bhargava, S. K.; Bennett, M. A. Coord. Chem. Rev. 2006, 250, 1851. (c) Steenwinkel, P.; Gossage, R. A.; van Koten, G. Chem. Eur. J. 1998, 4, 759. (d) Ryabov, A. D. Chem. Rev. 1990, 90, 403. such as 2-phenylpyridine, undergoes rapid and facile metalinduced $\mathrm{C}-\mathrm{H}$ bond activation at the ortho position adjacent to the pyridyl group, eventually affording a fairly stable, chelating interaction involving simultaneous formation of metal-nitrogen dative bonding and metal-carbon covalent bonding. It is notable that these heterocycles generally possess a highly delocalized $\pi$-system spreading across the whole chelate and the central transition-metal cation, which then exhibit unique photophysical properties compared to those of the free ligands. ${ }^{2}$

Certainly, the most well established cyclometalated heterocycles are 2-phenylpyridine and its functionalized deriva-

(2) Chou, P.-T.; Chi, Y. Chem. Eur. J. 2007, 13, 380.

Inorganic Chemistry, Vol. 47, No. 8, 20083307 
tives, which have been extensively used in synthetic organometallic chemistry because of their stable bidentate complexation with respect to a majority of late-transitionmetal elements. More specifically, 2-phenylpyridines are effective in forming stable cyclometalated complexes with the second-row transition-metal elements $\mathrm{Ru}(\mathrm{II}),{ }^{3} \mathrm{Rh}(\mathrm{III}),{ }^{4}$ and $\mathrm{Pd}(\mathrm{II})^{5}$ as well as the third-row transition-metal elements $\operatorname{Ir}(\mathrm{III})^{6}$ and $\mathrm{Pt}(\mathrm{II}){ }^{7}$ However, these ligands are rather inert to third-row transition-metal elements such as $\operatorname{Re}(\mathrm{I})$, possessing lower oxidation states. To our knowledge, only a few $\operatorname{Re}(\mathrm{I})$ complexes with cyclometalated ligands have ever been reported in the literature. ${ }^{8}$ Likewise, it was equally difficult to synthesize cyclometalated Os(II) metal complexes, except for a few cases in which the 2-phenylpyridine ligand could afford the anticipated five-membered chelation to the Os(II) metal cation. ${ }^{9}$ Interestingly, these Os(II) compounds were not prepared from the employment of an oxidative addition reaction but from an indirect metal exchange using bis(2-pyridyl)phenylmercury reagent. The reduced reactivity of aromatic heterocycles unfortunately hampered subsequent attempts for studying the photophysical properties, such as heavy-metal-induced spin-orbit coupling and the associated room-temperature phosphorescence, etc.

Recently, we have made assiduous efforts to design and synthesize several $\mathrm{Ru}(\mathrm{II})$ complexes possessing nitrogen heterocycles, including the aforementioned cyclometalated chelates, aimed at probing emission properties such as the energy gap, emission character, and quantum yield, i.e. the radiative versus the radiationless pathways, in correlation with their structural design. ${ }^{10}$ The second-row-metal complexes are well-suited for this approach, mainly because of the enhanced reactivity that is associated with ligand addition and substitution reactions. With regard to adverse effects, however, the weak metal-ligand bonding, in combination

(3) (a) Sasaki, I.; Vendier, L.; Sournia-Saquet, A.; Lacroix, P. G. Eur. J. Inorg. Chem. 2006, 3294. (b) Fraysse, S.; Coudret, C.; Launay, J.-P. J. Am. Chem. Soc. 2003, 125, 5880. (c) Hiraki, K.; Koizumi, M.; Kira, S.; Kawano, H. Chem. Lett. 1998, 47. (d) Patrick, J. M.; White, A. H.; Bruce, M. I.; Beatson, M. J.; Black, D. S. C.; Deacon, G. B.; Thomas, N. C. J. Chem. Soc., Dalton Trans. 1983, 2121.

(4) (a) Ghizdavu, L.; Lentzen, O.; Schumm, S.; Brodkorb, A.; Moucheron, C.; Kirsch-De Mesmaeker, A. Inorg. Chem. 2003, 42, 1935. (b) Lo, K. K.-W.; Li, C.-K.; Lau, K.-W.; Zhu, N. Dalton Trans. 2003, 4682. (c) Colombo, M. G.; Brunold, T. C.; Riedener, T.; Guedel, H. U.; Fortsch, M.; Buergi, H.-B. Inorg. Chem. 1994, 33, 545.

(5) (a) Hull, K. L.; Lanni, E. L.; Sanford, M. S. J. Am. Chem. Soc. 2006, 128, 14047. (b) Diez, A.; Fornies, J.; Garcia, A.; Lalinde, E.; Moreno, M. T. Inorg. Chem. 2005, 44, 2443. (c) Sanchez, G.; Garcia, J.; Meseguer, D.; Serrano, J. L.; Garcia, L.; Perez, J.; Lopez, G. Dalton Trans. 2003, 4709. (d) Ghedini, M.; Aiello, I.; La Deda, M.; Grisolia, A. Chem. Commun. 2003, 2198

(6) (a) Nazeeruddin, M. K.; Gratzel, M. Struct. Bonding (Berlin) 2007, 123, 113. (b) Flamigni, L.; Barbieri, A.; Sabatini, C.; Ventura, B.; Barigelletti, F. Top. Curr. Chem. 2007, 281, 143.

(7) (a) Sprouse, S.; King, K. A.; Spellane, P. J.; Watts, R. J. J. Am. Chem. Soc. 1984, 106, 6647. (b) Chassot, L.; Von Zelewsky, A. Inorg. Chem. 1987, 26, 2814. (c) Lu, W.; Mi, B.-X.; Chan, M. C. W.; Hui, Z.; Che, C.-M.; Zhu, N.; Lee, S.-T. J. Am. Chem. Soc. 2004, 126, 4958. (d) Koo, C.-K.; Ho, Y.-M.; Chow, C.-F.; Lam, M. H.-W.; Lau, T.-C.; Wong, W.-Y. Inorg. Chem. 2007, 46, 3603.

(8) (a) Vanhelmont, F. W. M.; Strouse, G. F.; Guedel, H. U.; Stueckl, A. C.; Schmalle, H. W. J. Phys. Chem. A 1997, 101, 2946. (b) Bruce, M. I.; Goodall, B. L.; Matsuda, I. Aust. J. Chem. 1975, 28, 1259.

(9) (a) Clark, A. M.; Rickard, C. E. F.; Roper, W. R.; Wright, L. J. Organometallics 1999, 18, 2813. (b) Ryabov, A. D.; Soukharev, V. S.; Alexandrova, L.; Le Lagadec, R.; Pfeffer, M. Inorg. Chem. 2003, 42, 6598. with a less pronounced heavy-atom effect, and hence the weaker spin-orbit coupling in cyclometalated $\mathrm{Ru}(\mathrm{II}) \mathrm{com}$ plexes have hampered their expansion in view of the inferior phosphorescent quantum yield: i.e., giving poor performances and reduced lifetime of the respective OLED devices. ${ }^{11}$ Alternatively, due to the stronger metal-ligand bonding, cyclometalated Os(II) complexes are expected to be more robust and inert than those of the second-row $\mathrm{Ru}(\mathrm{II})$ metal congeners, and it is thus critical to initiate a better synthetic method with the aim of probing the Os(II) metal analogues, including exploration of related photophysical characteristics from fundamental aspects. Particularly, we anticipated that incorporation of Os(II) elements would inevitably increase the energy of the metal-centered dd excited state (or ligand field (LF) transition) versus those of ligand-centered $\pi \pi^{*}$ or even the metal-to-ligand charge transfer (MLCT) transitions, giving phosphorescence with higher quantum yields. Detailed results concerning our approaches are elaborated in the following sections.

\section{Experimental Section}

2.1. General Information and Materials. Elemental analyses and mass spectra (operating in the FAB mode) were carried out at the NSC Regional Instrument Centre at National Chiao Tung University, Taiwan. ${ }^{1} \mathrm{H}$ and ${ }^{13} \mathrm{C}$ NMR spectra were recorded on a Varian Mercury $400 \mathrm{MHz}$ or an Inova $500 \mathrm{MHz}$ instrument; chemical shifts are quoted with respect to internal standard $\mathrm{Me}_{4} \mathrm{Si}$. All synthetic manipulations were performed under an $\mathrm{N}_{2}$ atmosphere, while solvents were used as received. The cyclometalated chelates 2-(2,4-difluorophenyl)pyridine (dfppyH) and 2-(2,4-bis(trifluoromethyl)phenyl)pyridine (btfppyH), were prepared by $\mathrm{Su}-$ zuki coupling employing 2-bromopyridine and the respective boronic acid according to the literature procedures. ${ }^{12}$

Steady-state absorption and emission spectra were recorded with a Hitachi (U-3310) spectrophotometer and an Edinburgh (FS920) fluorimeter, respectively. Solution samples, unless otherwise specified, were degassed by three freeze-pump - thaw cycles. Coumarin $480(\Phi=0.93$ in EtOH) and 4-(dicyanomethylene)-2-methyl-6( $p$-(dimethylamino)styryl)-4H-pyran (DCM; $\Phi=0.44$ in methanol $)^{13}$ were used as references to determine luminescence quantum yields of the studied compounds in solution, and the equation

$$
\Phi_{\mathrm{s}}=\Phi_{\mathrm{r}}\left(\frac{\eta_{\mathrm{s}}^{2} A_{\mathrm{r}} I_{\mathrm{s}}}{\eta_{\mathrm{r}}^{2} A_{\mathrm{s}} I_{\mathrm{r}}}\right)
$$

was used to calculate the emission quantum yields, where $\Phi_{\mathrm{s}}$ and $\Phi_{\mathrm{r}}$ are the quantum yields of the unknown and reference samples, $\eta$ is the refractive index of the solvent, $A_{\mathrm{r}}$ and $A_{\mathrm{s}}$ are the absorbances of the reference and the unknown samples at the excitation

(10) (a) Tung, Y.-L.; Lee, S.-W.; Chi, Y.; Chen, L.-S.; Shu, C.-F.; Wu, F.-I.; Carty, A. J.; Chou, P.-T.; Peng, S.-M.; Lee, G.-H. Adv. Mater. 2005, 17, 1059. (b) Tung, Y.-L.; Chen, L.-S.; Chi, Y.; Chou, P.-T.; Cheng, Y.-M.; Li, E. Y.; Lee, G.-H.; Shu, C.-F.; Wu, F.-I.; Carty, A. J. Adv. Funct. Mater. 2006, 16, 1615. (c) Chou, P.-T.; Chi, Y. Eur. J. Inorg. Chem. 2006, 3319.

(11) Li, E. Y.; Cheng, Y.-M.; Hsu, C.-C.; Chou, P.-T.; Lee, G.-H.; Lin, I.-H.; Chi, Y.; Liu, C.-S. Inorg. Chem. 2006, 45, 8041.

(12) (a) Beeby, A.; Bettington, S.; Fairlamb, I. J. S.; Goeta, A. E.; Kapdi, A. R.; Niemelae, E. H.; Thompson, A. L. New J. Chem. 2004, 28 , 600. (b) Coppo, P.; Plummer, E. A.; De Cola, L. Chem. Commun. 2004, 1774.

(13) Darke, M.; Lesiecki, M. L.; Camaioni, D. M. Chem. Phys. Lett. 1985, 113,530 . 
wavelength, and $I_{\mathrm{s}}$ and $I_{\mathrm{r}}$ are the integrated areas under the emission spectra of interest, respectively.

Lifetime studies were performed with an Edinburgh FL 900 photon counting system by using a hydrogen-filled or a nitrogen lamp as the excitation source. Data were analyzed by using the nonlinear least-squares procedure in combination with an iterative convolution method. The emission decays were analyzed by the sum of exponential functions, which allows partial removal of the instrument time broadening and consequently renders a temporal resolution of about 200 ps.

2.2. Preparation of $\left[\mathrm{Os}(\mathbf{p p y})_{2}(\mathbf{C O})_{2}\right](\mathbf{1 a}, \mathbf{b})$. In a $50 \mathrm{~mL}$ reaction flask, a mixture of $\mathrm{Os}_{3}(\mathrm{CO})_{12}(100 \mathrm{mg}, 0.11 \mathrm{mmol})$ and 2-phenylpyridine (ppy) $(106 \mathrm{mg}, 0.68 \mathrm{mmol})$ in $25 \mathrm{~mL}$ of diethylene glycol methyl ether (DGME) was heated to $180{ }^{\circ} \mathrm{C}$ for $24 \mathrm{~h}$. After the mixture was cooled to room temperature, the solvent was removed under vacuum and the residue was separated by silica gel column chromatography using a 1:4 mixture of ethyl acetate (EA) and hexane as eluent to give two isomeric complexes with the formula [Os(ppy $)_{2}(\mathrm{CO})_{2}$ ] (1a, $46 \mathrm{mg}, 0.083 \mathrm{mmol}, 25 \%$; 1b, 37 $\mathrm{mg}, 0.066 \mathrm{mmol}, 20 \%)$. Complexes 1a,b were further purified by recrystallization from a 1:2 mixture of EA and hexane and pure acetonitrile at room temperature, respectively.

2.2.1. Spectral Data for 1a. MS (FAB, $\left.{ }^{192} \mathrm{Os}\right): \mathrm{m} / \mathrm{z}, 556\left(\mathrm{M}^{+}\right)$, $528\left(\mathrm{M}^{+}-\mathrm{CO}\right), 500\left(\mathrm{M}^{+}-2 \mathrm{CO}\right)$. IR $\left(\mathrm{CH}_{2} \mathrm{Cl}_{2}\right): v(\mathrm{CO}) 1997$, $1924 \mathrm{~cm}^{-1} .{ }^{1} \mathrm{H}$ NMR $\left(500 \mathrm{MHz}\right.$, acetone- $\left.d_{6}, 298 \mathrm{~K}\right): \delta 8.20(\mathrm{dd}$, $2 \mathrm{H}, J=7.0,1.0 \mathrm{~Hz}), 8.09(\mathrm{~d}, 2 \mathrm{H}, J=8.5 \mathrm{~Hz}), 7.97(\mathrm{~d}, 2 \mathrm{H}, J=$ $8.0 \mathrm{~Hz}), 7.85-7.80(\mathrm{~m}, 4 \mathrm{H}), 7.33(\mathrm{td}, 2 \mathrm{H}, J=7.0,1.5 \mathrm{~Hz}), 7.21$ $(\mathrm{td}, 2 \mathrm{H}, J=7.2,1.5 \mathrm{~Hz}), 6.98$ (ddd, $2 \mathrm{H}, J=8.0,6.5,1.5 \mathrm{~Hz}$ ). Anal. Calcd for $\mathrm{C}_{24} \mathrm{H}_{16} \mathrm{~N}_{2} \mathrm{O}_{2} \mathrm{Os}$ : C, 51.97; H, 2.91; N, 5.05. Found: C, 51.86; H, 3.12; N, 5.05.

2.2.2. Spectral Data for 1b. MS (FAB, $\left.{ }^{192} \mathrm{Os}\right): \mathrm{m} / \mathrm{z}, 556\left(\mathrm{M}^{+}\right)$, $528\left(\mathrm{M}^{+}-\mathrm{CO}\right), 500\left(\mathrm{M}^{+}-2 \mathrm{CO}\right)$. IR $\left(\mathrm{CH}_{2} \mathrm{Cl}_{2}\right): v(\mathrm{CO}) 1993$, $1922 \mathrm{~cm}^{-1}$. ${ }^{1} \mathrm{H}$ NMR $\left(500 \mathrm{MHz}\right.$, acetone- $\left.d_{6}, 298 \mathrm{~K}\right): \delta 9.32(\mathrm{~d}$, $1 \mathrm{H}, J=5.5 \mathrm{~Hz}), 8.26(\mathrm{~d}, 1 \mathrm{H}, J=8.5 \mathrm{~Hz}), 8.16(\mathrm{td}, 1 \mathrm{H}, J=7.5$, $1.5 \mathrm{~Hz}), 8.01(\mathrm{t}, 2 \mathrm{H}, J=7.5 \mathrm{~Hz}), 7.91(\mathrm{~d}, 1 \mathrm{H}, J=7.5 \mathrm{~Hz})$, $7.81-7.76(\mathrm{~m}, 2 \mathrm{H}), 7.52(\mathrm{t}, 1 \mathrm{H}, J=6.0 \mathrm{~Hz}), 7.42(\mathrm{~d}, 1 \mathrm{H}, J=5.0$ $\mathrm{Hz}), 7.20(\mathrm{td}, 1 \mathrm{H}, J=6.5,2.0 \mathrm{~Hz}), 7.11(\mathrm{td}, 1 \mathrm{H}, J=8.0,2.0 \mathrm{~Hz})$, $6.98(\mathrm{td}, 1 \mathrm{H}, J=6.0,1.5 \mathrm{~Hz}), 6.89(\mathrm{td}, 1 \mathrm{H}, J=8.25,1.5 \mathrm{~Hz})$, $6.77(\mathrm{td}, 1 \mathrm{H}, J=8.0,1.0 \mathrm{~Hz}), 6.69(\mathrm{dd}, 1 \mathrm{H}, J=7.5,1.0 \mathrm{~Hz})$. Anal. Calcd for $\mathrm{C}_{24} \mathrm{H}_{16} \mathrm{~N}_{2} \mathrm{O}_{2}$ Os: C, 51.97; H, 2.91; N, 5.05. Found: C, 51.60; H, 2.94; N, 4.94 .

2.3. Preparation of $\left[\mathrm{Os}(\mathrm{dfppy})_{2}(\mathrm{CO})_{2}\right](2 \mathrm{a}, \mathrm{b})$ and $\left[\mathrm{Os}(\mathrm{btfppy})_{2-}\right.$ $(\mathbf{C O})_{2}$ ] (3a,b). The isomeric complexes [Os(dfppy $\left.)_{2}(\mathrm{CO})_{2}\right](\mathbf{2 a}$, $22 \% ; \mathbf{2} \mathbf{b}, 18 \%)$, and $\left[\mathrm{Os}(\text { btfppy })_{2}(\mathrm{CO})_{2}\right](\mathbf{3 a}, 4 \% ; \mathbf{3 b}, 6 \%)$, deriving from the respective reactions of $\mathrm{Os}_{3}(\mathrm{CO})_{12}$ with 2-(2,4-difluorophenyl)pyridine and with 2-(2,4-bis(trifluoromethyl)phenyl)pyridine, were obtained under similar conditions. Single crystals of $\mathbf{2 a}, \mathbf{b}$ suitable for X-ray diffraction studies were obtained from a 1:2 mixture of EA and hexane and anhydrous ethanol at room temperature, respectively.

2.3.1. Spectral Data for 2a. MS (FAB, $\left.{ }^{192} \mathrm{Os}\right): \mathrm{m} / \mathrm{z}, 628\left(\mathrm{M}^{+}\right)$, $572\left(\mathrm{M}^{+}-2 \mathrm{CO}\right)$. IR $\left(\mathrm{CH}_{2} \mathrm{Cl}_{2}\right): v(\mathrm{CO}) 2010,1940 \mathrm{~cm}^{-1} .{ }^{1} \mathrm{H} \mathrm{NMR}$ $\left(500 \mathrm{MHz}\right.$, acetone- $\left.d_{6}, 298 \mathrm{~K}\right): \delta 8.28(\mathrm{dd}, 2 \mathrm{H}, J=8.2,2.5 \mathrm{~Hz})$, $7.96-7.93(\mathrm{~m}, 4 \mathrm{H}), 7.70(\mathrm{dd}, 2 \mathrm{H}, J=7.5,2.5 \mathrm{~Hz}), 7.13(\mathrm{td}, 2 \mathrm{H}$, $J=7.0,1.0 \mathrm{~Hz}), 6.84-6.79(\mathrm{~m}, 2 \mathrm{H}) .{ }^{19} \mathrm{~F} \mathrm{NMR}\left(470 \mathrm{MHz}, \mathrm{CDCl}_{3}\right.$, $298 \mathrm{~K}): \delta-109.2(\mathrm{~s}, 2 \mathrm{~F}),-109.8(\mathrm{~s}, 2 \mathrm{~F})$. Anal. Calcd for $\mathrm{C}_{24} \mathrm{H}_{12} \mathrm{~F}_{4} \mathrm{~N}_{2} \mathrm{O}_{2} \mathrm{Os}$ : C, 46.00; H, 1.93; N, 4.47. Found: C, 46.08; H, 2.22; N, 4.56 .

2.3.2. Spectral Data for 2 b. MS (FAB, $\left.{ }^{192} \mathrm{Os}\right): \mathrm{m} / \mathrm{z}, 628\left(\mathrm{M}^{+}\right)$, $600\left(\mathrm{M}^{+}-\mathrm{CO}\right), 572\left(\mathrm{M}^{+}-2 \mathrm{CO}\right)$. IR $\left(\mathrm{CH}_{2} \mathrm{Cl}_{2}\right): v(\mathrm{CO}) 2006$, $1941 \mathrm{~cm}^{-1} .{ }^{1} \mathrm{H}$ NMR $\left(500 \mathrm{MHz}\right.$, acetone- $\left.d_{6}, 298 \mathrm{~K}\right): \delta 9.41(\mathrm{~d}$, $1 \mathrm{H}, J=5.5 \mathrm{~Hz}), 8.44(\mathrm{dd}, 1 \mathrm{H}, J=9.25,2.5 \mathrm{~Hz}), 8.28(\mathrm{dd}, 1 \mathrm{H}, J$ $=8.5,1.5 \mathrm{~Hz}), 8.24(\mathrm{t}, 1 \mathrm{H}, J=8.0 \mathrm{~Hz}), 7.91(\mathrm{t}, 1 \mathrm{H}, J=8.75 \mathrm{~Hz})$, $7.61-7.56(\mathrm{~m}, 2 \mathrm{H}), 7.51(\mathrm{~d}, 1 \mathrm{H}, J=5.2 \mathrm{~Hz}), 7.11(\mathrm{td}, 1 \mathrm{H}, J=$ 5.7, $1.0 \mathrm{~Hz}), 6.77(\mathrm{~m}, 1 \mathrm{H}), 6.54(\mathrm{~m}, 1 \mathrm{H}), 6.22(\mathrm{dd}, 1 \mathrm{H}, J=8.0$, $2.5 \mathrm{~Hz}) .{ }^{19} \mathrm{~F}$ NMR $\left(470 \mathrm{MHz}, \mathrm{CDCl}_{3}, 298 \mathrm{~K}\right): \delta-109.8(\mathrm{~s}, 1 \mathrm{~F})$, $-110.0(\mathrm{~s}, 1 \mathrm{~F}),-110.1(\mathrm{~s}, 1 \mathrm{~F}),-110.7$ (s, 1F). Anal. Calcd for $\mathrm{C}_{24} \mathrm{H}_{12} \mathrm{~F}_{4} \mathrm{~N}_{2} \mathrm{O}_{2} \mathrm{Os}$ : C, 46.00; H, 1.93; N, 4.47. Found: C, 45.87; H, $2.24 ; \mathrm{N}, 4.55$.

2.3.3. Spectral Data for 3a. MS (FAB, $\left.{ }^{192} \mathrm{Os}\right): m / z, 828\left(\mathrm{M}^{+}\right)$, $800\left(\mathrm{M}^{+}-\mathrm{CO}\right), 772\left(\mathrm{M}^{+}-2 \mathrm{CO}\right)$. IR $\left(\mathrm{CH}_{2} \mathrm{Cl}_{2}\right): v(\mathrm{CO}) 2014$, $1946 \mathrm{~cm}^{-1} .{ }^{1} \mathrm{H}$ NMR $\left(500 \mathrm{MHz}\right.$, acetone- $\left.d_{6}, 298 \mathrm{~K}\right): \delta 8.72(\mathrm{~s}$, $2 \mathrm{H}), 8.29$ (d, $2 \mathrm{H}, J=8.0 \mathrm{~Hz}), 8.09$ (ddd, $2 \mathrm{H}, J=8.2,6.0,2.5$ $\mathrm{Hz}), 7.96$ (dd, 2H, $J=6.0,1.0 \mathrm{~Hz}), 7.91$ (s, 2H), 7.27 (td, 2H, $J$ $=7.0,1.0 \mathrm{~Hz}) .{ }^{19} \mathrm{~F} \mathrm{NMR}\left(470 \mathrm{MHz}, \mathrm{CDCl}_{3}, 298 \mathrm{~K}\right): \delta-56.8(\mathrm{~s}$, $6 \mathrm{~F}$ ), -63.2 (s, 6F). Anal. Calcd for $\mathrm{C}_{28} \mathrm{H}_{12} \mathrm{~F}_{12} \mathrm{~N}_{2} \mathrm{O}_{2} \mathrm{Os}$ : C, 40.68; H, 1.46; N, 3.39. Found: C, 40.60; H, 1.93; N, 3.53 .

2.3.4. Spectral Data for 3b. MS (FAB, $\left.{ }^{192} \mathrm{Os}\right): \mathrm{m} / \mathrm{z}, 828\left(\mathrm{M}^{+}\right)$, $772\left(\mathrm{M}^{+}-2 \mathrm{CO}\right)$. IR $\left(\mathrm{CH}_{2} \mathrm{Cl}_{2}\right): v(\mathrm{CO}) 2010,1942 \mathrm{~cm}^{-1} .{ }^{1} \mathrm{H} \mathrm{NMR}$ $\left(500 \mathrm{MHz}\right.$, acetone- $\left.d_{6}, 298 \mathrm{~K}\right): \delta 9.60(\mathrm{dd}, 1 \mathrm{H}, J=5.5,1.0 \mathrm{~Hz})$, $8.59(\mathrm{~s}, 1 \mathrm{H}), 8.43(\mathrm{~d}, 1 \mathrm{H}, J=8.5 \mathrm{~Hz}), 8.39(\mathrm{td}, 1 \mathrm{H}, J=7.2,2.0$ $\mathrm{Hz}), 8.28(\mathrm{~d}, 1 \mathrm{H}, J=8.5 \mathrm{~Hz}), 8.06(\mathrm{td}, 1 \mathrm{H}, J=7.7,1.5 \mathrm{~Hz})$, $7.87-7.85(\mathrm{~m}, 2 \mathrm{H}), 7.78(\mathrm{td}, 1 \mathrm{H}, J=6.5,2.0 \mathrm{~Hz}), 7.61(\mathrm{~s}, 1 \mathrm{H})$, $7.32(\mathrm{td}, 1 \mathrm{H}, J=7.0,1.0 \mathrm{~Hz}), 6.83(\mathrm{~s}, 1 \mathrm{H}) .{ }^{19} \mathrm{~F} \mathrm{NMR}(470 \mathrm{MHz}$, $\left.\mathrm{CDCl}_{3}, 298 \mathrm{~K}\right): \delta-56.7(\mathrm{~s}, 3 \mathrm{~F}),-57.3(\mathrm{~s}, 3 \mathrm{~F}),-63.4(\mathrm{~s}, 3 \mathrm{~F})$, -64.2 (s, 3F). Anal. Calcd for $\mathrm{C}_{28} \mathrm{H}_{12} \mathrm{~F}_{12} \mathrm{~N}_{2} \mathrm{O}_{2} \mathrm{Os}$ : C, 40.68; H, 1.46; N, 3.39. Found: C, 40.39; H, 1.64; N, 3.42.

2.4. Phosphine Substitution. A stirred solution of $\mathbf{2 b}(50 \mathrm{mg}$, $0.08 \mathrm{mmol})$ in $15 \mathrm{~mL}$ of DGME was treated dropwise with a DGME solution $\left(3 \mathrm{~mL}\right.$ ) of freshly sublimed $\mathrm{Me}_{3} \mathrm{NO}(9 \mathrm{mg}, 0.12$ $\mathrm{mmol}$ ) at room temperature over a period of $5 \mathrm{~min}$. After that, the temperature was gradually raised to $100{ }^{\circ} \mathrm{C}$ within a period of 1 h. The phosphine ligand $\mathrm{PPh}_{2} \mathrm{Me}(48 \mathrm{mg}, 0.24 \mathrm{mmol})$ was added to this mixture, and the solution was further refluxed for $12 \mathrm{~h}$. After the mixture was cooled to room temperature, the solvent was evaporated, the residue was subjected to silica gel column chromatography, and the product was eluted with a 1:5 mixture of EA and hexane. The product $\left[\mathrm{Os}(\mathrm{dfppy})_{2}(\mathrm{CO})\left(\mathrm{PPh}_{2} \mathrm{Me}\right)\right]$ (4) was recrystallized from a mixture of EA and hexane to give yellow crystals $(26 \mathrm{mg}, 0.03 \mathrm{mmol}, 40 \%)$. The respective derivatives $\left[\mathrm{Os}(\mathrm{dfppy})_{2}(\mathrm{CO})\left(\mathrm{PPhMe}_{2}\right)\right](5)$ and $\left[\mathrm{Os}(\mathrm{btfppy})_{2}\left(\mathrm{PPh}_{2} \mathrm{Me}\right)(\mathrm{CO})\right](\mathbf{6})$ were prepared from the reaction of $\mathbf{2} \mathbf{b}$ with $\mathrm{PPhMe}_{2}$ and reactions of $\mathbf{3 a}$ (or $3 \mathbf{b}$ ) with $\mathrm{PPh}_{2} \mathrm{Me}$ in yields of 45 and $\sim 26 \%$, respectively.

2.4.1. Spectral Data for 4. MS (FAB, $\left.{ }^{192} \mathrm{Os}\right): \mathrm{m} / \mathrm{z} 800\left(\mathrm{M}^{+}\right)$. IR $\left(\mathrm{CH}_{2} \mathrm{Cl}_{2}\right): v(\mathrm{CO}) 1903 \mathrm{~cm}^{-1}$. ${ }^{1} \mathrm{H}$ NMR $\left(500 \mathrm{MHz}\right.$, acetone- $d_{6}, 298$ $\mathrm{K}): \delta 8.89(\mathrm{~d}, 1 \mathrm{H}, J=5.0 \mathrm{~Hz}), 8.38(\mathrm{dd}, 1 \mathrm{H}, J=8.25,2.5 \mathrm{~Hz})$, $8.04(\mathrm{t}, 1 \mathrm{H}, J=8.0 \mathrm{~Hz}), 7.99(\mathrm{dd}, 1 \mathrm{H}, J=8.5,2.5 \mathrm{~Hz}), 7.57(\mathrm{t}$, $1 \mathrm{H}, J=7.5 \mathrm{~Hz}), 7.48-7.44(\mathrm{~m}, 2 \mathrm{H}), 7.42-7.36(\mathrm{~m}, 3 \mathrm{H}), 7.30$ (dd, $1 \mathrm{H}, J=9.7,1.0 \mathrm{~Hz}), 7.24(\mathrm{~m}, 1 \mathrm{H}), 7.20-7.17(\mathrm{~m}, 4 \mathrm{H}), 7.14$ $(\mathrm{td}, 1 \mathrm{H}, J=7.0,1.0 \mathrm{~Hz}), 6.85(\mathrm{~d}, 1 \mathrm{H}, J=5.5 \mathrm{~Hz}), 6.67(\mathrm{t}, 1 \mathrm{H}, J$ $=6.0 \mathrm{~Hz}), 6.46-6.38(\mathrm{~m}, 3 \mathrm{H}), 1.61\left(\mathrm{~d}, 3 \mathrm{H}, J_{\mathrm{HP}}=8.0 \mathrm{~Hz}\right) .{ }^{19} \mathrm{~F}$ NMR (470 MHz, $\left.\mathrm{CDCl}_{3}, 298 \mathrm{~K}\right): \delta-111.0(\mathrm{~s}, 1 \mathrm{~F}),-111.7$ (s, 1F), $-111.8(\mathrm{~s}, 1 \mathrm{~F}),-112.5$ (s, 1F). ${ }^{31} \mathrm{P}$ NMR (202 MHz, acetone$\left.d_{6}, 298 \mathrm{~K}\right): \delta-4.5$ (s). Anal. Calcd for $\mathrm{C}_{36} \mathrm{H}_{25} \mathrm{~F}_{4} \mathrm{~N}_{2} \mathrm{OO}$ Os: C, 54.13; H, 3.15; N, 3.51. Found: C, 54.18; H, 3.45; N, 3.56 .

2.4.2. Spectral Data for 5. MS (FAB, $\left.{ }^{192} \mathrm{Os}\right): \mathrm{m} / \mathrm{z}, 739\left(\mathrm{M}^{+}+\right.$ 1). IR $\left(\mathrm{CH}_{2} \mathrm{Cl}_{2}\right): v(\mathrm{CO}) 1904 \mathrm{~cm}^{-1} .{ }^{1} \mathrm{H}$ NMR $(500 \mathrm{MHz}$, acetone$\left.d_{6}, 298 \mathrm{~K}\right): \delta 8.57(\mathrm{~d}, 1 \mathrm{H}, J=5.5 \mathrm{~Hz}), 8.32(\mathrm{dd}, 1 \mathrm{H}, J=8.2,3.0$ $\mathrm{Hz}), 8.14(\mathrm{dd}, 1 \mathrm{H}, J=8.5,2.0 \mathrm{~Hz}), 7.98(\mathrm{t}, 1 \mathrm{H}, J=8.0 \mathrm{~Hz}), 7.72$ $(\mathrm{t}, 1 \mathrm{H}, J=8.0 \mathrm{~Hz}), 7.56(\mathrm{dd}, 1 \mathrm{H}, J=9.0,1.0 \mathrm{~Hz}), 7.37-7.31(\mathrm{~m}$, $5 \mathrm{H}), 7.12(\mathrm{~d}, 1 \mathrm{H}, J=5.5 \mathrm{~Hz}), 7.07(\mathrm{td}, 1 \mathrm{H}, J=6.5,1.0 \mathrm{~Hz}), 6.88$ $(\mathrm{td}, 1 \mathrm{H}, J=7.0,1.5 \mathrm{~Hz}), 6.51(\mathrm{~m}, 1 \mathrm{H}), 6.39-6.34(\mathrm{~m}, 2 \mathrm{H}), 1.71$ $\left(\mathrm{d}, 3 \mathrm{H}, J_{\mathrm{HP}}=7.5 \mathrm{~Hz}\right), 1.18\left(\mathrm{~d}, 3 \mathrm{H}, J_{\mathrm{HP}}=7.5 \mathrm{~Hz}\right) .{ }^{19} \mathrm{~F}$ NMR $(470$ $\left.\mathrm{MHz}, \mathrm{CDCl}_{3}, 298 \mathrm{~K}\right): \delta-111.2$ (s, 1F), -111.5 (s, 1F), -111.9 
Table 1. Crystal Data and Refinement Parameters for Complexes 2a,b and 4

\begin{tabular}{llll}
\hline \multicolumn{1}{c}{ 2a } & \multicolumn{1}{c}{$\mathbf{2 b}$} & \multicolumn{1}{c}{$\mathbf{4}$} \\
\hline empirical formula & $\mathrm{C}_{24} \mathrm{H}_{12} \mathrm{~F}_{4} \mathrm{~N}_{2} \mathrm{O}_{2} \mathrm{Os}$ & $\mathrm{C}_{24} \mathrm{H}_{12} \mathrm{~F}_{4} \mathrm{~N}_{2} \mathrm{O}_{2} \mathrm{Os}$ & $\mathrm{C}_{36} \mathrm{H}_{25} \mathrm{~F}_{4} \mathrm{~N}_{2} \mathrm{OOss}$ \\
formula wt & 626.56 & 626.56 & 798.75 \\
temp, K & $150(2)$ & $150(2)$ & $295(2)$ \\
cryst syst & triclinic & monoclinic & monoclinic \\
Space group & $P \overline{1}$ & $P 21 / c$ & $P 21 n$ \\
$a, \AA$ & $10.8459(5)$ & $8.7294(5)$ & $8.7517(5)$ \\
$b, \AA$ & $13.1773(6)$ & $26.6052(16)$ & $17.2482(9)$ \\
$c, \AA$ & $15.3928(7)$ & $8.9896(5)$ & $19.8393(10)$
\end{tabular}

$\beta$, deg $\quad 71.845(1)$

$\gamma, \operatorname{deg} \quad 83.594(1)$

$V, \AA^{3} \quad 2018.06(16)$

$Z$

$\rho_{\text {calcd }}, \mathrm{g} \mathrm{cm}^{-3} \quad 2.062$

abs coeff, $\mathrm{mm}^{-1} \quad 6.381$

$F(000) \quad 1192$

cryst size, $\mathrm{mm}^{3} \quad 0.25 \times 0.18 \times 0.15$

no. of rflns collected 26873

no. of indep rflns $\quad 9222(R($ int $)=0.0362)$

max, min transmissn $\quad 0.4478,0.2984$

no. of data/restraints/params 9222/0/595

goodness-of-fit on $F^{2} \quad 1.041$

final $R$ indices $(I>2 \sigma(I)) \quad \mathrm{R} 1=0.0246, \mathrm{wR} 2=0.0518$

$R$ indices (all data) $\quad \mathrm{R} 1=0.0308, \mathrm{wR} 2=0.0544$

largest diff peak and hole, e $\AA^{-3} \quad 1.241$ and -0.693

$(\mathrm{s}, 1 \mathrm{~F}),-112.6(\mathrm{~s}, 1 \mathrm{~F}) .{ }^{31} \mathrm{P}$ NMR $\left(202 \mathrm{MHz}\right.$, acetone- $\left.d_{6}, 298 \mathrm{~K}\right)$ :

$\delta-26.2$ (s). Anal. Calcd for $\mathrm{C}_{31} \mathrm{H}_{23} \mathrm{~F}_{4} \mathrm{~N}_{2} \mathrm{OOsP}$ : C, 50.54; H, 3.15;

N, 3.80. Found: C, 49.89; H, 3.15; N, 3.73.

2.4.3. Spectral Data for 6. MS (FAB, $\left.{ }^{192} \mathrm{Os}\right): \mathrm{m} / \mathrm{z}, 1000\left(\mathrm{M}^{+}\right)$. IR $\left(\mathrm{CH}_{2} \mathrm{Cl}_{2}\right): v(\mathrm{CO}) 1910 \mathrm{~cm}^{-1} .{ }^{1} \mathrm{H}$ NMR $\left(500 \mathrm{MHz}\right.$, acetone- $d_{6}$, $298 \mathrm{~K}): \delta 9.05(\mathrm{~d}, 1 \mathrm{H}, J=6.25 \mathrm{~Hz}), 8.33(\mathrm{~d}, 1 \mathrm{H}, J=9.0 \mathrm{~Hz})$, $8.21(\mathrm{~m}, 1 \mathrm{H}), 8.18(\mathrm{~m}, 1 \mathrm{H}), 8.05(\mathrm{~d}, 1 \mathrm{H}, J=8.5 \mathrm{~Hz}), 7.74(\mathrm{t}, 1 \mathrm{H}$, $J=7.5 \mathrm{~Hz}), 7.48(\mathrm{~d}, 2 \mathrm{H}, J=7.5 \mathrm{~Hz}), 7.40-7.35(\mathrm{~m}, 3 \mathrm{H})$, $7.34-7.27(\mathrm{~m} \mathrm{8H}), 7.16(\mathrm{~s}, 1 \mathrm{H}), 7.06(\mathrm{~d}, 1 \mathrm{H}, J=5.5 \mathrm{~Hz}), 6.92(\mathrm{t}$, $1 \mathrm{H}, J=7.0 \mathrm{~Hz}), 1.60\left(\mathrm{~d}, 3 \mathrm{H}, J_{\mathrm{HP}}=7.5 \mathrm{~Hz}\right) .{ }^{19} \mathrm{~F} \mathrm{NMR}(470 \mathrm{MHz}$, $\left.\mathrm{CDCl}_{3}, 298 \mathrm{~K}\right): \delta-56.23(\mathrm{~s}, 3 \mathrm{~F}),-57.04(\mathrm{~s}, 3 \mathrm{~F}),-63.50(\mathrm{~s}, 3 \mathrm{~F})$, $-63.84(\mathrm{~s}, 3 \mathrm{~F}) .{ }^{31} \mathrm{P}$ NMR $\left(202 \mathrm{MHz}\right.$, acetone- $\left.d_{6}, 298 \mathrm{~K}\right): \delta-5.69$ (s). Anal. Calcd for $\mathrm{C}_{40} \mathrm{H}_{25} \mathrm{~F}_{12} \mathrm{~N}_{2} \mathrm{OOsP}$ : C, 48.10; H, 2.52; N, 2.80 . Found: C, 48.25; H, 2.91; N, 3.04.

2.5. X-ray Structural Measurements. Single-crystal X-ray data were measured on a Bruker SMART Apex CCD diffractometer using $\mu(\mathrm{Mo} \mathrm{K} \alpha)$ radiation $(\lambda=0.71073 \AA)$. The data collection was executed using the SMART program. Cell refinement and data reduction were carried out with the SAINT program. The structure was determined using the SHELXTL/PC program and refined using full-matrix least squares. All non-hydrogen atoms were refined anisotropically, whereas hydrogen atoms were placed at calculated positions and included in the final stage of refinements with fixed parameters. The crystal data and refinement parameters of $\mathbf{2 a , b a n d} \mathbf{4}$ are summarized in Table 1.

2.6. Theoretical Approach. Calculations on the electronic ground states of complexes $\mathbf{1}-\mathbf{6}$ were carried out by using B3LYP density functional theory. ${ }^{14} \mathrm{~A}$ "double- $\zeta$ " quality basis set consisting of the Hay and Wadt effective core potentials (LANL2DZ) ${ }^{15}$ was employed for the Os atom and 6-31G* basis $^{16}$ for the H, C, N, P, and $\mathrm{F}$ atoms. A relativistic effective core potential (ECP) replaced the inner core electrons of Os, leaving the outer core $\left(5 s^{2} 5 p^{6}\right)$ electrons and the $5 d^{6} 6 s^{2}$ valence electrons. Time-dependent DFT

(14) (a) Lee, C.; Yang, W.; Parr, R. G. Phys. Rev. B 1988, 37, 785. (b) Becke, A. D. J. Chem. Phys. 1993, 98, 5648.

(15) (a) Hay, P. J.; Wadt, R. W. J. Chem. Phys. 1985, 82, 270. (b) Wadt, W. R.; Hay, P. J. J. Chem. Phys. 1985, 82, 284. (c) Hay, P. J.; Wadt, W. R. J. Chem. Phys. 1985, 82, 299.

(16) Hariharan, P. C.; Pople, J. A. Mol. Phys. 1974, 27, 209.

3310 Inorganic Chemistry, Vol. 47, No. 8, 2008

$105.757(3)$

93.203(1)

$\begin{array}{ll}2009.4(2) & 2990.1(3) \\ 4 & 4 \\ 2.071 & 1.774 \\ 6.409 & 4.377 \\ 1192 & 1560 \\ 0.09 \times 0.26 \times 0.09 & 0.27 \times 0.10 \times 0.07 \\ 9858 & 19394 \\ 3495(R(\text { int })=0.0771) & 6874(R(\text { int })=0.0581) \\ 0.731 \text { and } 0.566 & 0.7492,0.3845 \\ 3495 / 0 / 298 & 6874 / 0 / 407 \\ 1.016 & 1.059 \\ R 1=0.0491, w R 2=0.0843 & \mathrm{R} 1=0.0432, \mathrm{wR} 2=0.0756 \\ \mathrm{R} 1=0.0997, \mathrm{wR} 2=0.0962 & \mathrm{R} 1=0.0615, \mathrm{wR} 2=0.0813 \\ 1.057 \text { and }-0.707 & 1.186 \text { and }-1.225\end{array}$

(TDDFT) calculations ${ }^{17}$ using the B3LYP functional were then performed on the basis of the structural optimized geometries. Typically, the lowest 10 triplet and 10 singlet roots of the nonhermitian eigenvalue equations were used to determine the vertical excitation energies. Oscillator strengths $(f)$ were deduced from the dipole transition matrix elements (for singlet states only). The ground-state B3LYP and excited-state TDDFT calculations were carried out by using Gaussian 03 , as described in our previous publications. ${ }^{18}$ The contribution of a group to a molecular orbital was calculated within the framework of Mulliken population analysis using the AOMix program. ${ }^{19}$

\section{Results}

3.1. Synthesis and Characterization. The syntheses of the anticipated Os(II) complexes started with the treatment of $\mathrm{Os}_{3}(\mathrm{CO})_{12}$ and 2-phenylpyridine or its functionalized derivatives in refluxing diethylene glycol methyl ether (DGME) solution. As described in Scheme 1, a nearly equal ratio of two isomeric products, denoted as $\mathbf{1 a}-\mathbf{3 a}$ and $\mathbf{1 b}-\mathbf{3 b}$, were obtained from these one-pot reactions in moderate yields. We also checked the distribution and relationship of products to show that the isomers $\mathbf{a}$ and $\mathbf{b}$ are the kinetic and thermodynamic products, respectively. Evidence in favor of such delineation comes from control experiments involving heating of a DGME solution of $\mathbf{1 a}$ (or 2a) at $180{ }^{\circ} \mathrm{C}$ for $24 \mathrm{~h}$, which afforded $\mathbf{1 a}, \mathbf{b}$ and $\mathbf{2 a , b}$ mixtures in ratios of 5:1 and 2:1, respectively, while heating of a solution of the thermodynamic isomer $\mathbf{1 b}$ (or $\mathbf{2 b}$ ) under

(17) (a) Jamorski, C.; Casida, M. E.; Salahub, D. R. J. Chem. Phys. 1996, 104, 5134. (b) Petersilka, M.; Grossmann, U. J.; Gross, E. K. U. Phys. Rev. Lett. 1996, 76, 1212. (c) Bauernschmitt, R.; Ahlrichs, R.; Hennrich, F. H.; Kappes, M. M. J. Am. Chem. Soc. 1998, 120, 5052. (d) Casida, M. E. J. Chem. Phys. 1998, 108, 4439. (e) Stratmann, R. E.; Scuseria, G. E.; Frisch, M. J. J. Chem. Phys. 1998, 109, 8218.

(18) (a) Yu, J.-K.; Cheng, Y.-M.; Hu, Y.-H.; Chou, P.-T.; Chen, Y.-L.; Lee, S.-W.; Chi, Y. J. Phys. Chem. B 2004, 108, 19908. (b) Chen, Y.-L.; Li, S.-W.; Chi, Y.; Cheng, Y.-M.; Pu, S.-C.; Yeh, Y.-S.; Chou, P.-T. ChemPhysChem 2005, 6, 2012.

(19) (a) Gorelsky, S. I. AOMix, revision 6.1; http://www.sg-chem.net/. (b) Gorelsky; S. I.; Lever, A. B. P. J. Organomet. Chem. 2001, 635, 187. 
Scheme 1

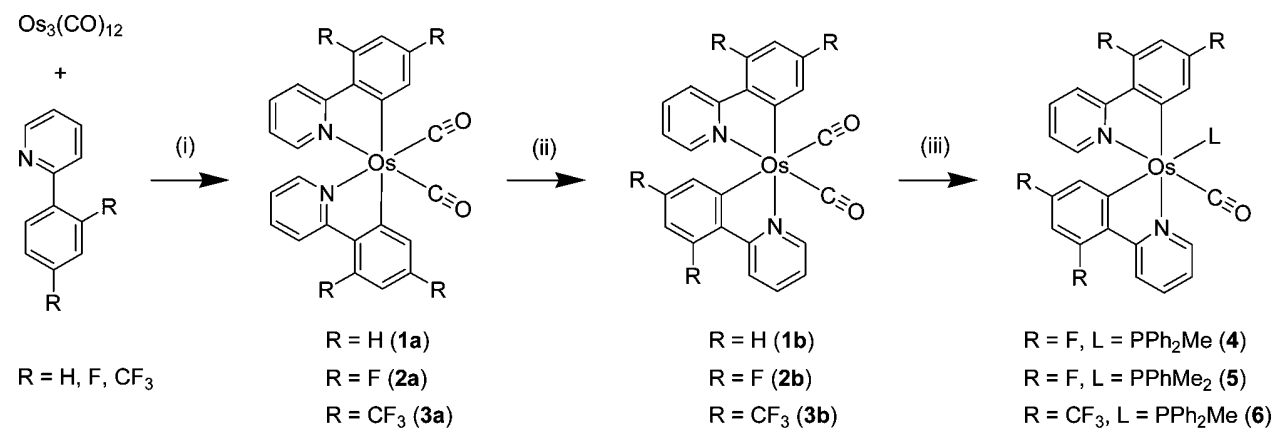

(i) DGME, $180^{\circ} \mathrm{C}, 24$ hours (ii) $180^{\circ} \mathrm{C}, 5 \sim 24$ hours (iii) 1.5 eq. $\mathrm{Me}_{3} \mathrm{NO}, 3$ eq. L, $180^{\circ} \mathrm{C}, 4$ hours

identical conditions afforded no reversion to the kinetic product 1a (or 2a). Moreover, complex 3a showed a much accelerated isomerization process during heating, giving a nearly 1:2 mixture of $\mathbf{3 a}, \mathbf{b}$ within a period of only $5 \mathrm{~h}$. Thus, it is reasonable to conclude that the electron-withdrawing substituents in these cyclometalated ligands lower the energy barrier and enhance the accompanying a-to-b isomerization.

The identity of these cyclometalated complexes was confirmed using ${ }^{1} \mathrm{H}$ NMR and IR spectroscopic techniques. For the a, b pair of complexes 1-3, comparison of the ${ }^{1} \mathrm{H}$ NMR spectra shows that the isomers a exhibited a much simpler spectral pattern due to the inherent molecular symmetry, i.e. the presence of a $C_{2}$ rotational axis, while the peaks corresponding to the isomers $\mathbf{b}$ are more complicated, confirming the lack of intrinsic symmetry, the result of which is in good agreement with the structural results of $\mathrm{X}$-ray diffraction studies (vide infra). On the other hand, IR analyses of all of these dicarbonyl complexes in solution gave two $v(\mathrm{CO})$ stretching bands of equal intensity, implying that the $\mathrm{CO}$ ligands adopt the cis orientation. It is also notable that the $v(\mathrm{CO})$ stretching frequencies of the $\mathbf{a}$, $\mathbf{b}$ pair of isomers $\mathbf{1} \mathbf{- 3}$ are shifted to a higher energy region by $13-19$ and $1-6 \mathrm{~cm}^{-1}$ upon changing the $\mathrm{R}$ substituents on the cyclometalated ligands from $\mathrm{H}$ to $\mathrm{F}$ and to $\mathrm{CF}_{3}$, respectively. This systematic shift indicates a continuous reduction of the electron density on the central Os(II) metal cation, leading to a decrease of the $\pi$-back-bonding to the CO ligands. Moreover, the $v(\mathrm{CO})$ stretching bands of isomer $\mathbf{a}$ in all complexes 1-3 are shifted to higher energy by a maximum of $4 \mathrm{~cm}^{-1}$ versus those of their $\mathbf{b}$ counterparts. This observation is consistent with an increase of electron density at the Os(II) metal center of isomers b related to $\mathbf{a}$. Moreover, this observation is in good agreement with the increased metal $\mathrm{d}_{\pi}$ contribution to the HOMO of all isomers $\mathbf{b}$, as well as the occurrence of excessive $\pi$-back-bonding to only one $\mathrm{CO}$ ligand in $\mathbf{b}$ according to the result of DFT calculations (see discussion elaborated in Theoretical Investigations).

Single-crystal X-ray diffraction studies on $\mathbf{2 a}, \mathbf{b}$ have been conducted to reveal their structural features. Figure 1 depicts the ORTEP diagram of 2a, showing a slightly distorted octahedral arrangement, with two cis-oriented CO ligands, two cis pyridine fragments, and two mutually trans-aligned 4,6-difluorophenyl groups. In sharp contrast, the dfppy ligands in $\mathbf{2 b}$ show a distinctive arrangement of chelating ligands on the Os(II) center (Figure 2). More precisely, although the orientation of its cis-CO ligands remains unaltered, the carbon donor of one cyclometalated ligand now

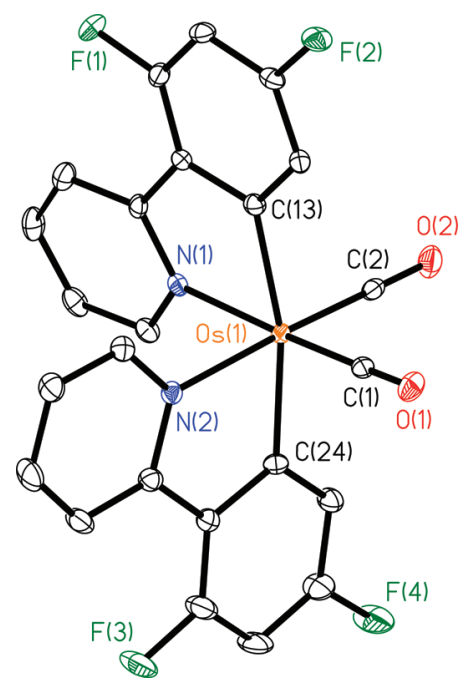

Figure 1. ORTEP drawing of complex 2a with thermal ellipsoids shown at the $50 \%$ probability level. Selected distances $(\AA)$ : Os(1)-C(1)=1.864(4), $\mathrm{Os}(1)-\mathrm{C}(2)=1.859(4), \mathrm{Os}(1)-\mathrm{C}(13)=2.116(3), \mathrm{Os}(1)-\mathrm{C}(24)=2.110(3)$, $\mathrm{Os}(1)-\mathrm{N}(1)=2.145(3), \mathrm{Os}(1)-\mathrm{N}(2)=2.146(3), \mathrm{O}(1)-\mathrm{C}(1)=1.145(4)$, $\mathrm{O}(2)-\mathrm{C}(2)=1.158(4)$.

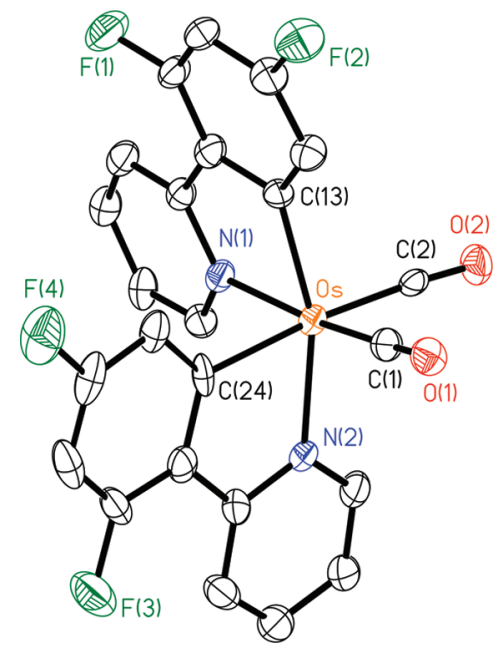

Figure 2. ORTEP drawing of complex $\mathbf{2 b}$ with thermal ellipsoids shown at the $50 \%$ probability level. Selected distances $(\AA)$ : Os $-\mathrm{C}(1)=1.865(11)$, $\mathrm{Os}-\mathrm{C}(2)=1.940(12), \mathrm{Os}-\mathrm{C}(13)=2.078(9), \mathrm{Os}-\mathrm{C}(24)=2.129(9)$, $\mathrm{Os}-\mathrm{N}(1)=2.142(7), \mathrm{Os}-\mathrm{N}(2)=2.159(8), \mathrm{O}(1)-\mathrm{C}(1)=1.155(11)$, $\mathrm{O}(2)-\mathrm{C}(2)=1.137(11)$. 
resides trans to the nitrogen atom of the second dfppy ligand, showing that isomer $\mathbf{2 b}$ is related to $\mathbf{2} \mathbf{a}$ by a $180^{\circ}$ rotation of one cyclometalated ligand with respect to the central Os(II) atom. The symmetric ligand arrangement shown by $\mathbf{2 a}$ has been observed in several cyclometalated $\mathrm{Ru}$ (II) complexes documented in the literature $;^{20}$ however, the asymmetrical arrangement of chelates shown by $\mathbf{2 b}$ is far less common, particularly for the system involving two cis-oriented $\mathrm{CO}$ ligands. $^{21}$

After confirming their crystal structure, we then proceeded to investigate their chemical reactivity. In one approach, the anticipated phosphine substitution products exhibit enhanced emission quantum efficiencies at room temperature. This is mainly due to the fact that phosphine showed reduced $\pi$-accepting abilities and hence destabilized the metal $\mathrm{d}_{\pi}$ orbitals, resulting in an increased proportion of MLCT contribution to the lowest energy excited state. Accordingly, these reactions were conducted via a stepwise manner, involving a prior treatment with freshly sublimed $\mathrm{Me}_{3} \mathrm{NO}$, followed by addition of phosphine. ${ }^{22}$ The mixture was then generally stirred at $\sim 180{ }^{\circ} \mathrm{C}$ for $12 \mathrm{~h}$, and the product was isolated by routine manipulation.

Remarkably, the ppy complexes 1a,b failed to afford any substitution product under all attempts. This could be due to the higher electron density located at the $\mathrm{CO}$ ligands, which is confirmed by its relatively lower $v(\mathrm{CO})$ stretching bands shown in the IR spectra. The excessive electron density on the CO ligands would then inhibit the reaction with the nucleophilic $\mathrm{Me}_{3} \mathrm{NO}$ reagent. ${ }^{23}$ On the other hand, the dfppy complex $\mathbf{2 b}$, due to its reduced electron density at the Os(II) center, showed a much improved reactivity versus its ppy counterpart $\mathbf{1 b}$, giving rise to the required phosphine adducts $\left[\mathrm{Os}(\mathrm{dfppy})_{2}(\mathrm{CO})\left(\mathrm{PPh}_{2} \mathrm{Me}\right)\right](\mathbf{4})$ and $\left[\mathrm{Os}(\mathrm{dfppy})_{2^{-}}\right.$ $\left.(\mathrm{CO})\left(\mathrm{PPhMe}_{2}\right)\right](5)$ in moderate yields, while the respective isomer $\mathbf{2 a}$ is inert to the added phosphine under similar conditions. Figure 3 depicts the ORTEP diagram of $\mathbf{4}$, showing that the $\mathrm{PPh}_{2} \mathrm{Me}$ indeed replaced the $\mathrm{CO}$ occupying the site trans to the carbon atom of one cyclometalated chelate of $\mathbf{2 b}$. The result shows good agreement with the selective weakening of one Os-CO linkage in $\mathbf{2 b}$, for which the necessary trans labilization effect is provided by the stronger metal-carbon covalent bonding. It is notable that the incoming phosphine and the remaining $\mathrm{CO}$ ligand in $\mathbf{4}$ adopt the cis arrangement, which is in sharp contrast to that of the reaction product of the dicarbonyl complex $\left[\mathrm{Os}(\mathrm{fppz})_{2}(\mathrm{CO})_{2}\right]$ with pyridine, the latter afforded the transsubstituted [Os(fppz $\left.)_{2}(\mathrm{CO})(\mathrm{py})\right]$ (fppz $=3$-(trifluoromethyl)5-(2-pyridyl)pyrazolate) in high yield. ${ }^{24}$ Finally, for the btfppy complexes 3a,b, due to their increased electron

(20) Zhang, Q.-F.; Cheung, K.-M.; Williams, I. D.; Leung, W.-H. Eur. J. Inorg. Chem. 2005, 4780.

(21) (a) Lai, Y.-H.; Chou, T.-Y.; Song, Y.-H.; Liu, C.-S.; Chi, Y.; Carty, A. J.; Peng, S.-M.; Lee, G.-H. Chem. Mater. 2003, 15, 2454. (b) Chen, Y.-L.; Lee, S.-W.; Chi, Y.; Hwang, K.-C.; Kumar, S. B.; Hu, Y.-H.; Cheng, Y.-M.; Chou, P.-T.; Peng, S.-M.; Lee, G.-H.; Yeh, S.-J.; Chen, C.-T. Inorg. Chem. 2005, 44, 4287.

(22) Tung, Y.-L.; Lee, S.-W.; Chi, Y.; Tao, Y.-T.; Chien, C.-H.; Cheng, Y.-M.; Chou, P.-T.; Peng, S.-M.; Liu, C.-S. J. Mater. Chem. 2005, 15,460 .

(23) Luh, T. Y. Coord. Chem. Rev. 1984, 60, 255.

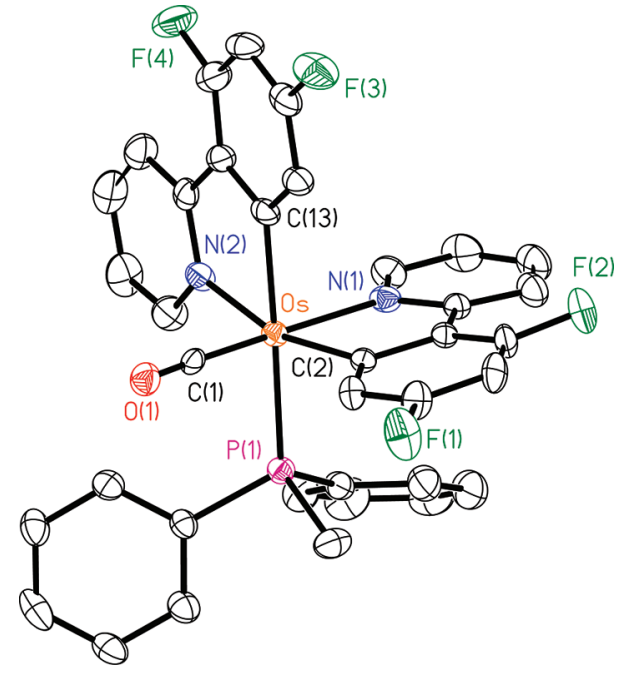

Figure 3. ORTEP drawing of complex $\mathbf{4}$ with thermal ellipsoids shown at the $30 \%$ probability level. Selected distances $(\AA)$ : Os $-\mathrm{C}(1)=1.826(6)$, $\mathrm{Os}-\mathrm{C}(2)=2.041(5), \quad \mathrm{Os}-\mathrm{N}(1)=2.151(4), \mathrm{Os}-\mathrm{N}(2)=2.170(4)$, $\mathrm{Os}-\mathrm{C}(13)=2.098(5), \mathrm{Os}-\mathrm{P}(1)=2.369(14), \mathrm{O}(1)-\mathrm{C}(1)=1.166(6)$.

deficiency at the Os(II) metal center and much faster isomerization rate from $\mathbf{3 a}$ to $\mathbf{3 b}$ (vide supra), both isomers can readily react with $\mathrm{PPh}_{2} \mathrm{Me}$ to give the single adduct $\left[\mathrm{Os}(\text { btfppy })_{2}(\mathrm{CO})\left(\mathrm{PPh}_{2} \mathrm{Me}\right)\right]$ (6). It is also notable that complex $\mathbf{6}$ is isolated in lower yields versus those for $\mathbf{4}$ and $\mathbf{5}$, which could be due to the subsequent sample decomposition induced by the possible consecutive loss of the second CO ligand.

3.2. Photophysical Properties. Figure 4 illustrates the absorption and emission spectra of all of the titled complexes 1-6 in dichloromethane, while Table 2 summarizes the important photophysical parameters. In general, $\mathbf{1}-\mathbf{6}$ possess allowed absorption bands in the UV region of $<320 \mathrm{~nm}$, for which $\epsilon$ values at the absorption maxima were calculated to be $>10^{4} \mathrm{M}^{-1} \mathrm{~cm}^{-1}$ and can thus be attributed to the local ${ }^{1} \pi \pi^{*}$ transition of the heterocyclic chelates. Next, the lowerlying absorption band with peak wavelengths at 360-485 $\mathrm{nm}$ can be reasonably assigned to a spin-allowed metal-toligand charge transfer (MLCT) transition in the singlet manifold. It is notable that all isomers $\mathbf{b}$ showed the ${ }^{1} \mathrm{MLCT}$ absorption being red-shifted from that of the respective isomers $\mathbf{a}$. This is consistent with the greater electron density at the Os(II) metal center of isomers $\mathbf{b}$, revealed by their lowered $v(\mathrm{CO})$ stretching bands observed in the infrared spectra (vide infra).

In addition, the lowest energy absorption of both isomers of $\mathbf{3}$ occurred at the longest wavelength region and 1-3 exhibited the sequence $\mathbf{3}>\mathbf{1}>\mathbf{2}$ : $\mathrm{cf}$. 3a $(375 \mathrm{~nm})>\mathbf{1 a}$ $(365 \mathrm{~nm})>\mathbf{2 a}(345 \mathrm{~nm})$ and $\mathbf{3 b}(400 \mathrm{~nm})>\mathbf{1 b}(382 \mathrm{~nm})$ $>\mathbf{2 b}(363 \mathrm{~nm})$. This observation is attributed to the fact that the $\mathrm{CF}_{3}$ substituents of complexes $\mathbf{3}$ are highly electron withdrawing via inductive effects and would naturally lower the energy of the $\pi^{*}$ orbitals on the adjacent pyridyl group as well as the $\mathrm{d}_{\pi}$ orbital on the metal center. If the stabilization energy on the pyridyl $\pi^{*}$ orbital is relatively

(24) Li, S.-W.; Cheng, Y.-M.; Yeh, Y.-S.; Hsu, C.-C.; Chou, P.-T.; Peng, S.-M.; Lee, G.-H.; Tung, Y.-L.; Wu, P.-C.; Chi, Y.; Wu, F.-I.; Shu, C.-F. Chem. Eur. J. 2005, 11, 6347. 

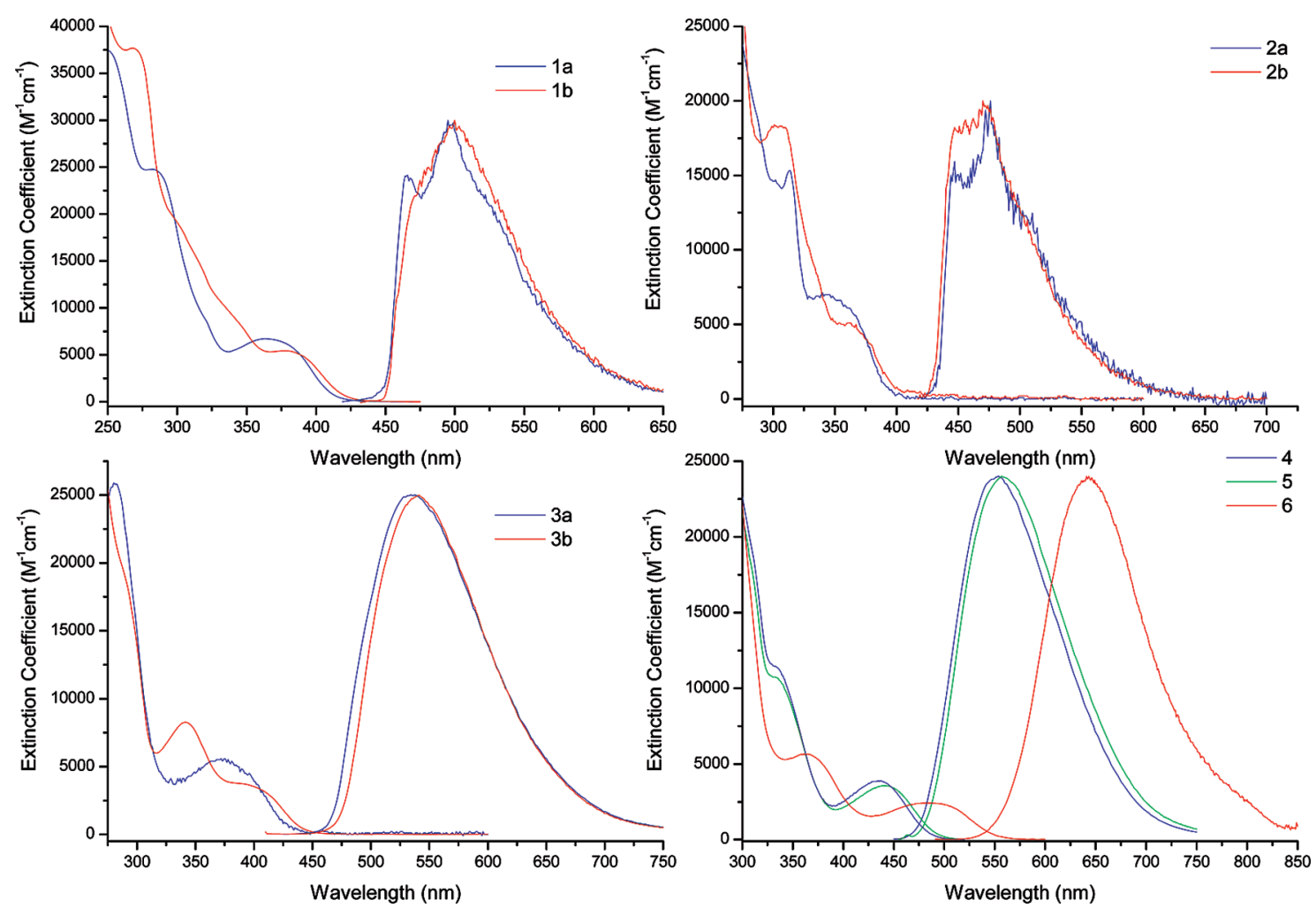

Figure 4. UV/vis absorption and normalized emission spectra of all $\mathrm{Os}(\mathrm{II})$ complexes in $\mathrm{CH}_{2} \mathrm{Cl}_{2}(298 \mathrm{~K})$.

Table 2. Photophysical and Electrochemical Data for All Os(II) Metal Complexes

\begin{tabular}{|c|c|c|c|c|c|c|c|c|}
\hline & UV-vis/nm $\left(\epsilon \times 10^{-3}\right)$ & PL $\lambda_{\max }(\mathrm{nm})^{a}$ & QY & $\tau_{\mathrm{obs}}(\mu \mathrm{s})$ & $k_{\mathrm{r}}\left(\mathrm{s}^{-1}\right)^{b}$ & $k_{\mathrm{nr}}\left(\mathrm{s}^{-1}\right)$ & $E_{1 / 2}^{\mathrm{ox}}(\mathrm{V})^{c}$ & $E_{1 / 2}{ }^{\text {red }}(\mathrm{V})^{c}$ \\
\hline $1 \mathbf{a}$ & 285 (24.7), 365 (6.7) & 466,499 & 0.0008 & 0.014 & $5.7 \times 10^{4}$ & $7.1 \times 10^{7}$ & $0.80^{d}$ & $-2.56,-2.84$ \\
\hline 1b & $270(37.6), 382(5.4)$ & 500 & 0.0005 & 0.006 & $8.3 \times 10^{4}$ & $1.7 \times 10^{8}$ & $0.71^{d}$ & $-2.58,-2.84$ \\
\hline $2 \mathbf{a}$ & $283(20.3), 314(15.3), 345(6.9)$ & 447,476 & 0.0006 & 0.012 & $5.0 \times 10^{4}$ & $8.3 \times 10^{7}$ & $1.12^{d}$ & $-2.42,-2.75$ \\
\hline $3 \mathbf{a}$ & $282(25.8), 375(5.6)$ & 535 & 0.18 & 3.06 & $5.9 \times 10^{4}$ & $2.7 \times 10^{5}$ & $1.26^{d}$ & \\
\hline $3 \mathbf{b}$ & $288(19.5), 342(8.3), 400(3.5)$ & 541 & 0.20 & 1.44 & $1.4 \times 10^{5}$ & $5.6 \times 10^{5}$ & $1.17^{d}$ & \\
\hline 4 & $311(18.6), 332(11.4), 435(3.9)$ & 553 & 0.15 & 0.32 & $4.7 \times 10^{5}$ & $2.7 \times 10^{6}$ & 0.33 & $-2.63,-3.10$ \\
\hline
\end{tabular}

${ }^{a} \mathrm{UV}$-vis spectra were recorded in $\mathrm{CH}_{2} \mathrm{Cl}_{2}$, while $\mathrm{PL}$ measurements were conducted in degassed $\mathrm{CH}_{2} \mathrm{Cl}_{2}$ solution at room temperature. ${ }^{b} k_{\mathrm{r}}=\mathrm{QY} / \tau_{\mathrm{obs}} ; k_{\mathrm{nr}}$ $=\left(1 / \tau_{\text {obs }}\right)-k_{\mathrm{r}} \cdot{ }^{c}$ Potentials were quoted versus the $\mathrm{Fc} / \mathrm{Fc}^{+}$reference; oxidation and reduction potentials were measured in $\mathrm{CH}_{2} \mathrm{Cl}_{2}$ and $\mathrm{THF}$, respectively. ${ }^{d}$ Anodic peak potential.

greater than that imposed on the Os(II) $\mathrm{d}_{\pi}$ orbitals, a substantial amount of red shift would be expected for their ${ }^{1}$ MLCT transitions, consistent with the experimentally observed trend. As for both isomers of $\mathbf{2}$, a greater stabilization of the metal $\mathrm{d}_{\pi}$ orbitals is expected, as the electronwithdrawing fluoro substituents are located at the meta positions with respect to the Os(II) metal center. On the other hand, since these fluoro substituents are also located at the positions ortho and para to the pyridyl group, there is no doubt that the resonance (mesomeric) effects would exert an influence opposite to the anticipated inductive effect, ${ }^{25}$ giving a much reduced influence on the energy level of the adjacent pyridyl $\pi^{*}$ orbital, and showing a blue-shifted absorption that is substantially higher in energy than that of their parent ppy complexes $\mathbf{1}$. Although such properties have not been explicitly mentioned in the literature, ${ }^{6 \mathrm{a}}$ the respec-

(25) Cheng, Y.-M.; Yeh, Y.-S.; Ho, M.-L.; Chou, P.-T.; Chen, P.-S.; Chi, Y. Inorg. Chem. 2005, 44, 4594. tive dfppy chelates were successfully utilized for the preparation of many blue phosphorescent complexes, by taking advantage of its higher lying $\pi^{*}$ orbital on the pyridyl segment. ${ }^{26}$

The replacement of one of the carbonyl group with a phosphine ligand in complexes $\mathbf{4}-\mathbf{6}$ further shifts the peak wavelength to lower energy as compared to those of $\mathbf{2}$ and 3. These results can be rationalized by the increase of $d_{\pi}$ orbital energy pertaining to the Os(II) metal atom and thereby decreasing the energy gaps of the ${ }^{1}$ MLCT transitions in these

(26) (a) Coppo, P.; Plummer, E. A.; De Cola, L. Chem. Commun. 2004, 1774. (b) Tamayo, A. B.; Alleyne, B. D.; Djurovich, P. I.; Lamansky, S.; Tsyba, I.; Ho, N. N.; Bau, R.; Thompson, M. E. J. Am. Chem. Soc. 2003, 125, 7377. (c) Li, J.; Djurovich, P. I.; Alleyne, B. D.; Yousufuddin, M.; Ho, N. N.; Thomas, J. C.; Peters, J. C.; Bau, R.; Thompson, M. E. Inorg. Chem. 2005, 44, 1713. (d) Wu, L.-L.; Yang, C.-H.; Sun, I.-W.; Chu, S.-Y.; Kao, P.-C.; Huang, H.-H. Organometallics 2007, 26, 2017. (e) Yang, C.-H.; Cheng, Y.-M.; Chi, Y.; Hsu, C.-J.; Fang, F.-C.; Wong, K.-T.; Chou, P.-T.; Chang, C.-H.; Tsai, M.H.; Wu, C.-C. Angew. Chem., Int. Ed. 2007, 46, 2418. (f) Yeh, S.-J.; Wu, W.-C.; Chen, C.-T.; Song, Y.-H.; Chi, Y.; Ho, M.-H.; Hsu, S.F.; Chen, C.-H. Adv. Mater. 2005, 17, 285. 
phosphine adducts. Moreover, the absorption peak of $\mathbf{6}$ occurring at a rather long wavelength of $485 \mathrm{~nm}$ is mainly attributed to the significant stabilization of the $\pi^{*}$ orbital (on the pyridyl moiety) imposed by the two $\mathrm{CF}_{3}$ substituents (on the phenyl group) through the inductive effect.

Examination of the photoluminescence upon excitation at $355 \mathrm{~nm}$ reveals a remarkable correlation of their photophysical properties to the functional groups on the cyclometalated ligands. The parent ppy complexes 1a,b showed extremely weak luminescence at room temperature, possibly due to the emission derived in principle from the typical ligand-centered $\pi \pi^{*}$ excited state. This hypothesis was further confirmed by an emission featuring rather distinct vibronic progressions, implying less contribution from the characteristic MLCT. Conversely, the difluoro substituents of $\mathbf{2 a}, \mathbf{b}$ lowered the energy of the occupied ligand $\pi$ orbital and consequently improved the mixing with the Os(II) metal $d_{\pi}$ orbitals. However, their emissions were still dominated by the $\pi \pi^{*}$ transition, as confirmed qualitatively by the vibronic progression detected. Following the same trend, the substitution of the strongest electron-withdrawing $\mathrm{CF}_{3}$ groups, forming $\mathbf{3 a}, \mathbf{b}$, further lowered the occupied ligand $\pi$-orbitals with respect to the metal $\mathrm{d}_{\pi}$ orbitals, resulting in an increase of the MLCT contribution to the lowest lying transition. From the steadystate approach, this delineation is supported by the lack of vibronic coupling and observation of only featureless emission profile in solution at room temperature.

For the $\mathrm{PPh}_{2} \mathrm{Me}$-substituted complexes $\mathbf{4}$ and $\mathbf{6}$, a bathochromic shift of emission versus their dicarbonyl counterparts was notably observed: cf. $2 \mathbf{b}(472 \mathrm{~nm}), \mathbf{3 b}(541 \mathrm{~nm})$ and $\mathbf{4}$ $(553 \mathrm{~nm}), 6(642 \mathrm{~nm})$. This is apparently caused by a decrease of the $\pi$-accepting strength of $\mathrm{PPh}_{2} \mathrm{Me}$, which in turn pushes up the energy of the metal $\mathrm{d}_{\pi}$ level. Moreover, upon the introduction of an even less $\pi$-accepting phosphine such as $\mathrm{PPhMe}_{2}$, we obtained complex 5, which exhibited emission at $557 \mathrm{~nm}$. The variation in emission gap, i.e., peak wavelength, between $\mathbf{4}$ and $\mathbf{5}$ seems lower than those expected on the basis of the electronic properties of the phosphine ligands. ${ }^{27}$ Details of this discussion will be presented in Theoretical Investigations.

3.3. Electrochemistry. The electrochemical behavior of these Os(II) metal complexes was investigated by cyclic voltammetry using ferrocene as the internal standard. The respective redox data are also given in Table 2. During the anodic scan in $\mathrm{CH}_{2} \mathrm{Cl}_{2}$, all dicarbonyl Os(II) complexes $\mathbf{1}-\mathbf{3}$ exhibited either an irreversible or a quasi-reversible oxidation at the metal center, with anodic peak potentials according to the ascending sequences $\mathbf{1}<\mathbf{2}<\mathbf{3}$ and $\mathbf{b}<\mathbf{a}$. These trends are consistent with the electron-withdrawing effect of the fluorine and $\mathrm{CF}_{3}$ substituents, which stabilized the metal-centered $\mathrm{d}_{\pi}$ orbitals, as well as the solution IR $v(\mathrm{CO})$ data, which reveal the greater electron-deficient character for the a isomers. Upon switching to the cathodic sweep in THF,

(27) (a) Vogler, A.; Kunkely, H. Coord. Chem. Rev. 2002, 230, 243. (b) Tung, Y.-L.; Wu, P.-C.; Liu, C.-S.; Chi, Y.; Yu, J.-K.; Hu, Y.-H.; Chou, P.-T.; Peng, S.-M.; Lee, G.-H.; Tao, Y.; Carty, A. J.; Shu, C.F.; Wu, F.-I. Organometallics 2004, 23, 3745. (c) Tsubaki, H.; Tohyama, S.; Koike, K.; Saitoh, H.; Ishitani, O. Dalton Trans. 2005, 385 . two reversible reduction processes, with potentials ranging from -2.56 to $-2.42 \mathrm{~V}$ and -2.84 to $-2.74 \mathrm{~V}$, were detected for complexes $\mathbf{1}$ and $\mathbf{2}$, showing that the reductions occur on each of the cyclometalated ligands and that the reduction potentials decrease upon switching from phenyl to the difluorophenyl group. Unfortunately, the corresponding reduction potentials for complexes $\mathbf{3 a}, \mathbf{b}$ cannot be seen, which prohibits a further comparison of the electrochemical data.

Similarly, the redox potentials of the phosphine-substituted Os(II) complexes 4-6 are also notably affected by the cyclometalated ligands. This was revealed by the fact that all redox potentials of $\mathbf{6}$ are much greater than those of $\mathbf{4}$, versus the smaller difference noted between $\mathbf{4}$ and $\mathbf{5}$, which agrees with the minor disturbance upon varying the phosphine ligand. Moreover, it should be noted that a smaller change of oxidation versus the reduction potentials was observed between $\mathbf{4}$ and $\mathbf{6}$, and this is consistent with the fact that the $\mathrm{CF}_{3}$ substituents possessed a relatively reduced electron-withdrawing effect to the Os(II) metal atom.

3.4. Theoretical Investigations. To gain insight into the photophysical behavior of all of the title complexes, density functional theory (DFT) was applied to molecular orbital studies. Figure 5 depicts the unoccupied and occupied frontier orbitals of $\mathbf{2}$ and $\mathbf{3}$ that are mainly involved in the lowestlying transitions. Description and details of the energy gap of each transition of all complexes are given in Table 3. Clearly, if one neglects the associated vibronic and solvation broadening effects, the calculated lowest triplet states are close in energy to the $0-0$ onset of phosphorescence obtained experimentally. For instance, the $\mathrm{S}_{0}-\mathrm{T}_{1}$ energy gaps, which are calculated to be 428 and $463 \mathrm{~nm}$ for $\mathbf{2 a}$ and $\mathbf{3 a}$, respectively, are in agreement with the experimental results of $\sim 430 \mathrm{~nm}$ (2a) and $\sim 470 \mathrm{~nm}$ (3a). Therefore, the theoretical level adopted is well justified for interpreting the photophysical properties of the complexes concerned here. As depicted in Figure 5, the lowest-lying electronic transition $\left(\mathrm{S}_{1}\right.$ and $\mathrm{T}_{1}$ ) of complexes $\mathbf{2}$ and $\mathbf{3}$ is composed of MLCT $\left(\mathrm{d}_{\pi} \rightarrow\right.$ pyridyl fragment) mixed with a certain extent of intraligand charge transfer (ILCT, for a isomers) or ligandto-ligand charge transfer (LLCT for $\mathbf{b}$ isomers, i.e. substituted phenyl $\rightarrow$ pyridyl fragment). Furthermore, the contribution of MLCT to the lowest lying transitions $\left(S_{1}\right.$ and $\left.T_{1}\right)$ in $\mathbf{b}$ isomers, which serves as the key factor in promoting spin-orbit coupling, is calculated to be greater than that of the corresponding a isomers. Moreover, as for a isomers, the contribution of MLCT to the lowest lying transitions $\left(\mathrm{T}_{1}\right)$ was calculated to be in the order 3a $(35.5 \%)>\mathbf{1 a}(25.7 \%)$ $>\mathbf{2 a}(20.4 \%)$. Likewise, $\mathbf{b}$ isomers reveal a similar ordering: 3b $(38.6 \%)>$ 1b $(30.4 \%)>$ 2b $(29.9 \%)$. These results correlate well with the conclusion drawn from the radiative decay rate constants given in Table 2 and have been rationalized in terms of the substituent effects on the HOMO and/or LUMO associated with $\pi, \pi^{*}$, or $\mathrm{d}_{\pi}$ electron density (vide supra).

It is also interesting to note that the contribution of $\mathrm{CO}$ fragments in HOMO is non-negligible and the electron density distributions among the two carbonyl fragments in 


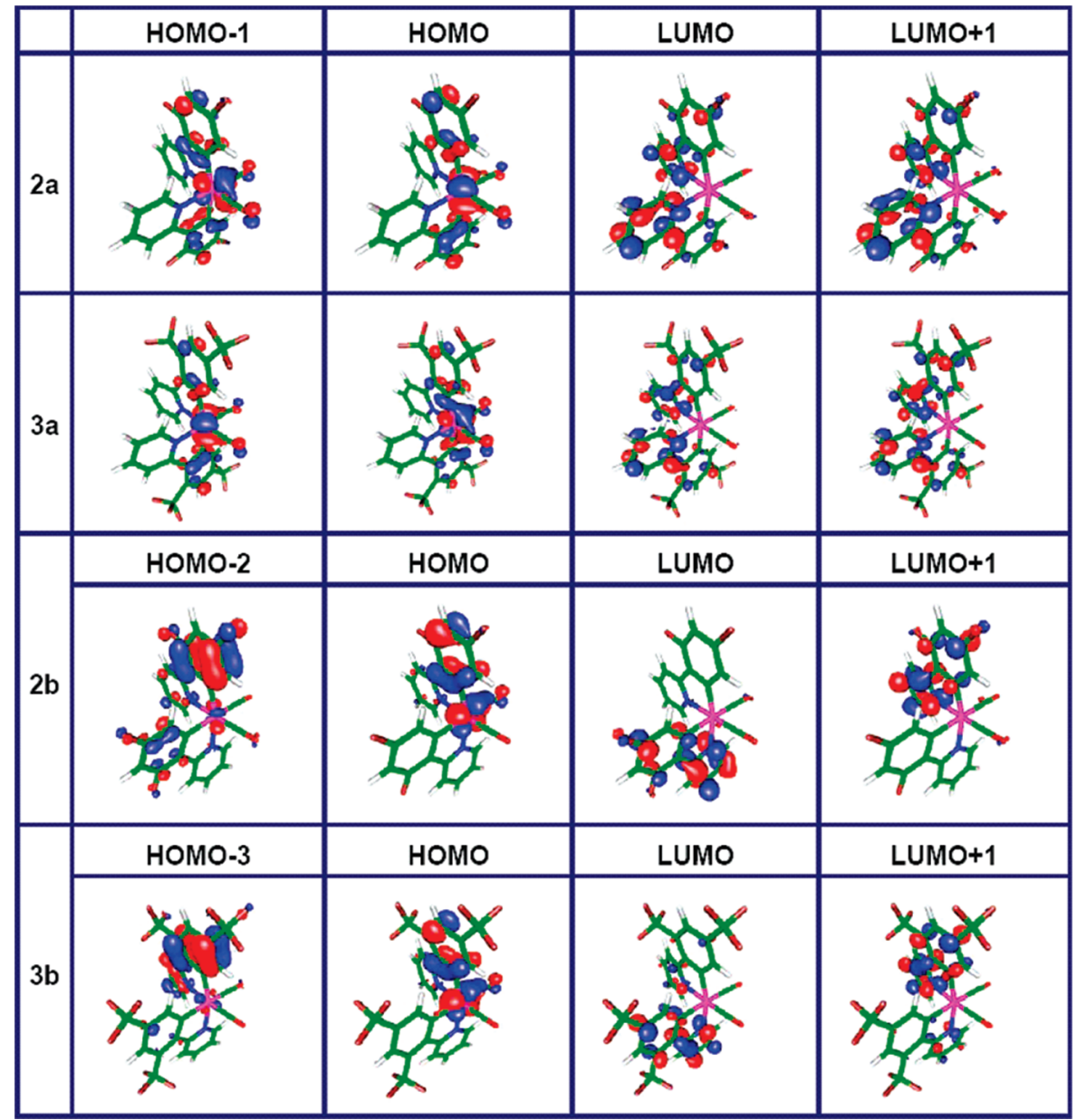

Figure 5. Frontier orbitals involved in the lowest lying singlet and triplet excited states for complexes $\mathbf{2}$ and $\mathbf{3}$.

Table 3. Calculated Energy Levels and Orbital Transition Analyses of All of the Title Complexes

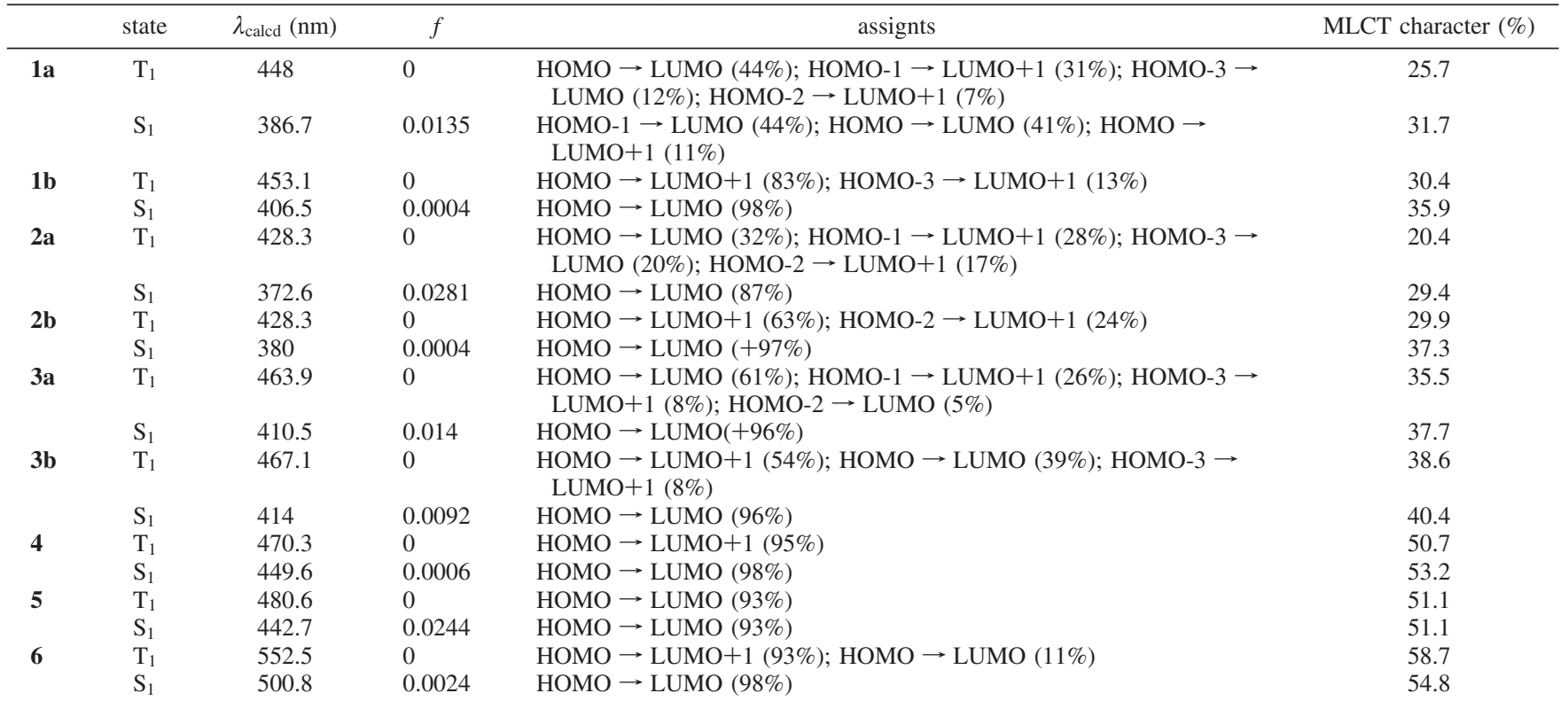

HOMO is more or less equal in the a isomers, while the electron density in $\mathbf{b}$ isomers is excessively located in one of the carbonyl fragments, which is in trans orientation to the phenyl fragment (see Figure 5). The difference in the electron density distribution is plausibly caused by a symmetry issue, in which a isomers are more symmetrical than b in view of the ligand orientations. Since the $S_{0} \rightarrow T_{1}$ transition, in part, involves the shift of electron density from $\mathrm{CO}$ to pyridyl moiety, the result of which might cause the weakening of the Os(II)-CO bond in different degree. ${ }^{28}$ Although direct evidence is still lacking we believe that 


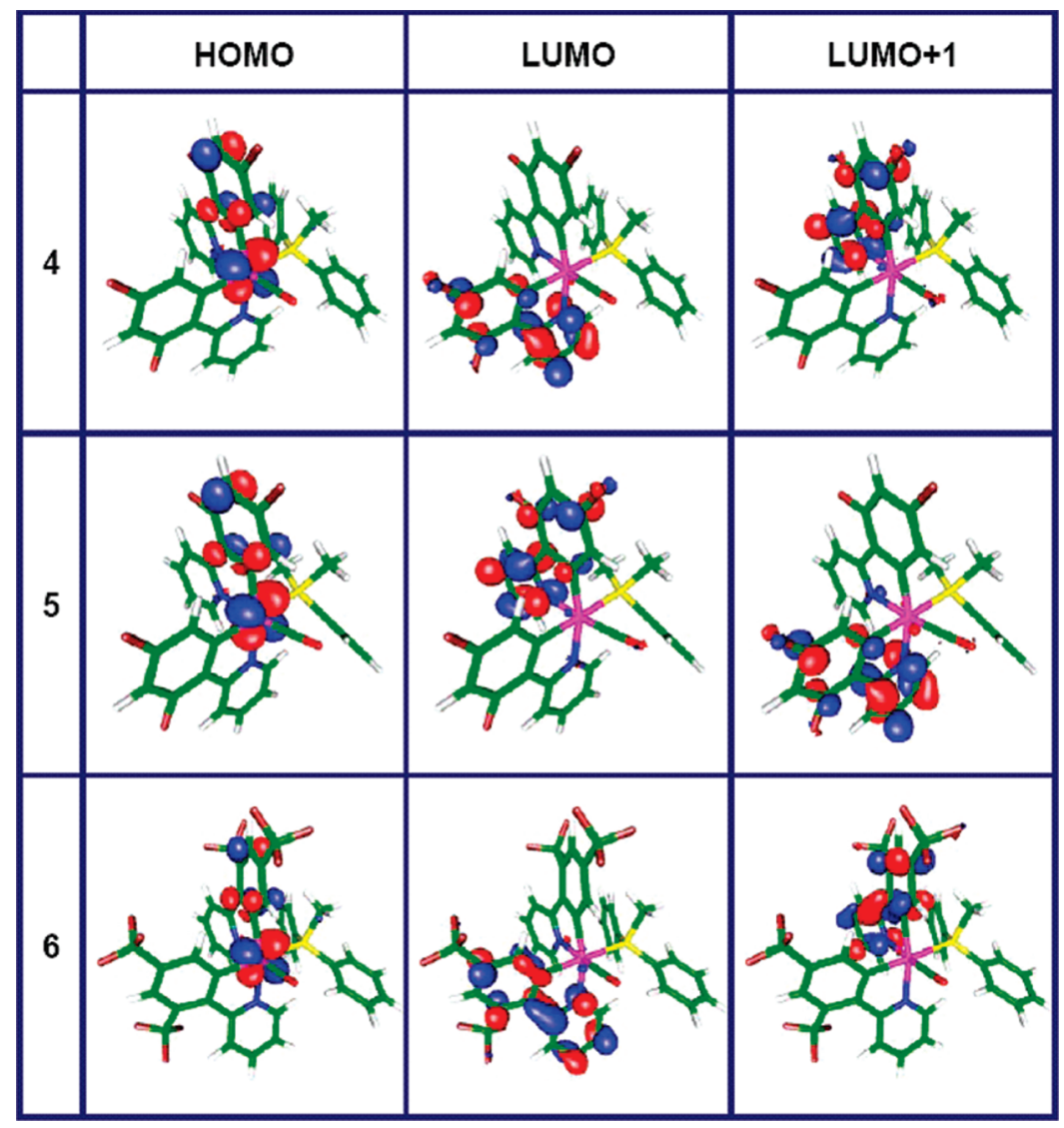

Figure 6. Frontier orbitals involved in the lowest lying singlet and triplet excited states for complexes 4-6.

this subtle variation may be partially responsible to the larger nonradiative rate constants for the $\mathbf{b}$ isomers (see Table 2).

In the case of complexes $\mathbf{4}$ and $\mathbf{5}$, their lower-lying transitions, i.e. the $S_{1}$ (or $T_{1}$ ) state, share the same pattern $(\mathrm{HOMO} \rightarrow$ LUMO) of electronic orbital transition, in which the electron densities are all excited from the difluorophenyl fragment to the pyridyl part of the same or the other cyclometalated ligand mixed, to a great extent, with an MLCT transition (see Figure 6). For example, the MLCT contributions to the $\mathrm{S}_{0} \rightarrow \mathrm{T}_{1}$ transitions of complexes $\mathbf{4}$ and 5 are $50.7 \%$ and $51.1 \%$, respectively, which are significantly greater than those of $\mathbf{1}-\mathbf{3}$ (see Table 3), justifying the fact that $\mathrm{PPh}_{2} \mathrm{Me}$ (or $\mathrm{PPhMe}_{2}$ ) has less $\pi$ accepting power than that of CO. Moreover, the replacement of one phenyl group on the $\mathrm{PPh}_{2} \mathrm{Me}$ ligand site (4) to a methyl group (5) results in a slight red shift of the calculated $\mathrm{S}_{0}-\mathrm{T}_{1}$ gap (see Table $3)$. The results are consistent with the experimental observations in terms of emission peak wavelengths and can be rationalized by the reduced $\pi$ accepting strength for $\mathrm{PPhMe}_{2}$ (vide supra). Despite the moderate red shift of phosphorescence from 4 to $\mathbf{5}$, a salient difference lies in the emission yield of 0.15 (4) versus unity (5) in degassed $\mathrm{CH}_{2} \mathrm{Cl}_{2}$. While the radiative decay rate constant is about equal between $\mathbf{4}$ $\left(4.7 \times 10^{5} \mathrm{~s}^{-1}\right)$ and $\mathbf{5}\left(4.0 \times 10^{5} \mathrm{~s}^{-1}\right)$, the major difference

(28) (a) Hsu, F.-C.; Tung, Y.-L.; Chi, Y.; Hsu, C.-C.; Cheng, Y.-M.; Ho, M.-L.; Chou, P.-T.; Peng, S.-M.; Carty, A. J. Inorg. Chem. 2006, 45, 10188. (b) Cheng, Y.-M.; Li, E. Y.; Lee, G.-H.; Chou, P.-T.; Lin, S.-Y.; Shu, C.-F.; Hwang, K.-C.; Chen, Y.-L.; Song, Y.-H.; Chi, Y. Inorg. Chem. 2007, 46, 10276.
Table 4. Contributions of All Fragment Orbitals to the Molecular Orbitals Which Are Involved in the $\mathrm{S}_{0} \rightarrow \mathrm{S}_{1}$ (or $\mathrm{T}_{1}$ ) Transitions

\begin{tabular}{lrrrrrr}
\hline & \multicolumn{2}{c}{$\mathbf{4}$} & & \multicolumn{3}{c}{$\mathbf{6}$} \\
\cline { 2 - 3 } & $\begin{array}{c}\text { HOMO } \\
(\%)\end{array}$ & $\begin{array}{c}\text { LUMO } \\
(\%)\end{array}$ & & $\begin{array}{c}\text { HOMO } \\
(\%)\end{array}$ & $\begin{array}{c}\text { LUMO } \\
(\%)\end{array}$ & $\begin{array}{c}\text { LUMO+1 } \\
(\%)\end{array}$ \\
\hline Os atom & 56.8 & 2.5 & & 58.8 & 2.9 & 2.3 \\
carbonyl & 0.3 & 1.5 & & 0.4 & 0.4 & 1.2 \\
PPh ${ }_{2}$ Me & 4.9 & 1.0 & & 3.8 & 0.5 & 1.7 \\
phenyl parts & 33.0 & 18.9 & & 31.3 & 38.7 & 43.1 \\
pyridyl parts & 5.0 & 76.1 & & 5.7 & 57.5 & 51.7
\end{tabular}

seems to be due to the difference in the nonradiative decay rate constants of $2.7 \times 10^{6} \mathrm{~s}^{-1}$ and $<4 \times 10^{3} \mathrm{~s}^{-1}$ for $\mathbf{4}$ and 5 , respectively. Though pending resolution, we tentatively attribute this difference to more vibrational degrees of freedom for $\mathrm{PPh}_{2} \mathrm{Me}$ (4) than for $\mathrm{PPhMe}_{2}(5)$, such that the accessible deactivation channels are expected to be greater in 4. This viewpoint can be further supported by the relatively low quantum yield (0.04) in $\mathbf{6}$, bearing the $\mathrm{PPh}_{2} \mathrm{Me}$ ligand. However, replacing the fluoride in $\mathbf{4}$ by a $\mathrm{CF}_{3}$ functional group, forming $\mathbf{6}$, sharply decreases the calculated energy gap as compared to that of $\mathbf{4}$ and $\mathbf{5}$. As for the rationalization, Table 4 shows the contributions of all fragment orbitals to the molecular orbitals that are involved in the $S_{0} \rightarrow T_{1}$ transition. Obviously, the contributions of the phenyl fragments to the HOMO for $\mathbf{4}$ and $\mathbf{6}$ are similar, while the electron density on the phenyl fragments turns out to be $\sim 2$ fold larger in the LUMO for 6 as compared to 4 . One therefore envisages that the orbital energy of the $\pi^{*}$ orbitals of pyridyl fragments in $\mathbf{4}$ is higher than that in $\mathbf{6}$. Qualitatively, this can be rationalized by the resonance (mesomeric) 
effects invoked by the fluoro substituents located at the positions ortho and para to the pyridyl group (vide supra). This conclusion is consistent with the experimentally observed greatly red-shifted phosphorescence $(642 \mathrm{~nm})$ in 6 and is reminiscent of the blue-shifted phosphorescence for $\mathbf{2}$ as compared to that of $\mathbf{3}$.

\section{Conclusion}

In conclusion, a series of new Os(II) complexes bearing cyclometalated phenylpyridine ligands have been designed and synthesized with the aim to investigate the associated photoluminescence properties. Our results demonstrate the correlation between MLCT and radiative lifetime and hence the efficiency of radiationless deactivation. Complexes $\mathbf{1 a}$ (2a) and $\mathbf{1 b}(\mathbf{2} \mathbf{b})$ possess a great extent of ${ }^{3} \pi \pi^{*}$ character and long radiative lifetimes. This, in combination with perhaps an increase in deactivating modes, gives rise to an essentially nonemissive (very weak emission) property at room temperature. Upon the substitution of electronwithdrawing fluoro and $\mathrm{CF}_{3}$ groups on the phenyl group of chelates, a progressive decrease of $\pi \pi^{*}$ and an increase in
MLCT characters were noted. A further increase of MLCT character was achieved by substituting phosphine for the $\mathrm{CO}$ ligand, forming highly efficient green and red phosphorescent Os(II) complexes. Under the premise of this proposed mechanism, one may be able to analyze the lower lying electronic transitions and the corresponding frontier orbital analyses, providing a guideline to predict not only the phosphorescence properties such as peak wavelength and transition properties but also the quantum efficiency in a qualitative manner. We thus believe that the results presented here may be of great importance in the design of luminescence materials incorporating Os(II) metal elements.

Acknowledgment. We are grateful for financial support from the National Science Council of Taiwan.

Supporting Information Available: CIF files giving X-ray crystallographic data for complexes $\mathbf{2 a}, \mathbf{b}$ and $\mathbf{4}$. This material is available free of charge via the Internet at http://pubs.acs.org.

IC7023132 